\title{
Analysis of MIMO Systems in the Presence of Co-channel Interference and Spatial Correlation
}

\author{
Dian-Wu Yue and Qian Wang \\ Dalian Maritime University \\ China
}

\section{Introduction}

Wireless communication systems employing multiple antenna elements at the transmitter and the receiver have been attracting much interest in recent years due to the significant capacity gain promised by the multiple-input multiple-output (MIMO) systems [Teletar (1999)], [Foschini \& Gans (1998)]. The MIMO systems have been analyzed deeply from two different perspectives [Teletar (1999)]-[Yue \& Zhang (2010)]: one concerns the evaluation of the information-theoretic (Shannon) capacity, the other concerns performance evaluation in terms of outage probability or symbol error probability of practical systems. Both of the capacity analysis and performance analysis strongly rely on random matrix theory and matrix variate distributions.

So far the capacity issues of MIMO systems have been extensively studied in the literature, yet with main focus on the scenario without interference [Teletar (1999)]-[Kiessling (2005)]. In cellular systems, however, multiple users share the same radio spectrum, which typically causes co-channel interference. It is well known that co-channel interference ultimately limits the quality of service offered to the users. There have been initial investigations for the MIMO capacity with co-channel interference in fading environments [Catreux et al. (2000)]-[Kang \& Alouini (2003a)]. In particular, Song and Blostein [Song \& Blostein (2002)] studied the behavior of MIMO capacity with varying number of interferers through simulations. In [Kang et al. (2007)] and [Kang \& Alouini (2003a)], Kang et al. obtained exact closed-form expressions for the moment generating function, mean, variance of MIMO capacity. Specifically, the paper [Kang et al. (2007)] considered MIMO Rayleigh fading channels in the presence of additive noise and interferers with arbitrary average powers, but requiring that there is no spatial correlation both among transmit antenna elements and among receive antenna elements for the desired user, and there is no correlation among receive antenna elements for any interferer. Reference [Kang \& Alouini (2003a)] considered MIMO Rician channels in the presence of Rayleigh co-channel interference, but requiring that there is an identical correlation structure among receive antenna elements for both the desired user and interferers, and there is no correlation among their transmit antenna elements.

In many practical situations, however, signal correlation among the antenna elements exists in realistic environments due to poor scattering conditions. A typical example of this is an uplink transmission from a mobile station (MS) to a base station (BS), where around the 
BS the number of scatterers is usually smaller than around mobile terminals, thus leading to the fact that the correlation at the BS is stronger than at the MS. Another typical example of this is a downlink transmission from a BS to a MS, where the antennas at the BS can be spaced sufficiently far to achieve uncorrelation among them. On the other hand, it is more difficult to space the antennas far apart at the mobile terminals due to physical size constraints, and consequently correlation arises among the antenna elements in such scenarios. The above factors have given us an impetus for studying the capacity of MIMO channels with interference and receive correlation [Wang \& Yue (2009)]. In Section 3, we will investigate the capacity issue in the case where the MIMO channels of the desired user and co-channel interferers are all subject to Rayleigh type of fading.

A MIMO system can be configured differently. One configuration is transmit/receive diversity (TRD) which has been widely used due to its simplicity and good performance. The performance of MIMO systems with optimal TRD depends on their operational environments. Their performance in a Rayleigh fading environment without co-channel interference was investigated by Dighe et al. [Dighe et al. (2001)] by assuming that the MIMO channels follow independent and identical (i.i.d.) Rayleigh distribution. The resulting outage probability is expressible in the form of a determinant. This result was subsequently extended by Kang and Alouini [Kang \& Alouini (2003b)] to a general case of independent, but not necessarily identically distributed, Rician fading channels. The results, again, takes the form of determinants. For the case using dual antennas at the transmitter or receiver end, they obtained [Kang \& Alouini (2004a)] an explicit expression for outage probability complementing the result of Dighe [Dighe et al. (2001)]. The performance of MIMO systems with optimal TRD in the presence of co-channel interference was tackled in [Dighe et al. (2003)] and [Kang \& Alouini (2004b)] under various fading environments allowing for the MIMO fading channels of the intended user and interferers to be non-i.i.d. Rician/Rayleigh, i.i.d. Rician/Rayleigh, and Rayleigh/Rayleigh. All these studies focus on MIMO systems with uncorrelated or semi-correlated antennas.

By semi-correlation, we mean that the spatial correlation exists only at one side, transmitter or receiver end, of the MIMO systems. Even for the case with semi-correlation, it is usually assumed that the intended user and interferers have the same correlation structure to simplify the mathematical analysis. In fact, the use of this assumption leads to the same mathematical treatment as the one with i.i.d. channels. The i.i.d. or uncorrelated assumption is often invalid in many practical applications. Significant correlation among the antennas exists in realistic environments due to, for example, limited spacing between antennas. Furthermore, the spatial structure (and even the fading distribution) of the interference usually differ from its counterpart for the intended user since their signals propagate over different multi-paths, suffer from different fading, and arrive at the receive antenna array with different incident angles. To handle these general fading situations, we must take different methodology [Yue \& Zhang (2010)]. In Section 4, we will investigate the performance issue of MIMO systems with optimal TRD mainly over general Rayleigh/Rayleigh fading channels in a unified framework. Throughout the paper, we use extensively relevant notations and results from multivariate statistical theory, in particular, various matrix-variate distributions. Although relevant results are available in the statistical literature [Muirhead (1982)], [Gupta \& Nagar (2000)] and [Mathai et al. (1995)], they are given only for real variables. The extension of these results to their complex counterparts, as required in this paper, is straightforward. Such results, though useful for wireless communications, are not found in the open references. We therefore first 
summarize definitions of various special functions with complex matrix arguments and their properties in Section 2 for the use in this paper which, we hope, are also useful to researchers in the area of wireless communications.

Moreover, we will use the following notations throughout the paper. By $\mathbf{I}_{n}$ we denote the identity matrix of size $n \times n$ (the subscript will be omitted wherever the size of the matrix is clear from the context), $\mathbf{0}$ signifies the all-zero matrix, $\operatorname{diag}\left(x_{1}, \ldots, x_{n}\right)$ denotes the diagonal matrix with elements $x_{1}, \ldots, x_{n}$, the determinant of the matrix $\mathbf{X}$ is denoted by $|\mathbf{X}| \operatorname{or} \operatorname{det}(\mathbf{X})$, $\left[x_{i j}\right]$ is a matrix with $x_{i j}$ representing its $(i, j)$ th element and correspondingly, $\left|x_{i j}\right|$ denotes its determinant. eig $(\mathbf{X})$ denotes the diagonal matrix of eigenvalues of $\mathbf{X}$. The symbol $\mathbf{X}>0$ indicates that $\mathbf{X}$ is positive definite; likewise, $\mathbf{X}>\mathbf{A}$ means $\mathbf{X}-\mathbf{A}>0$. We use notation $\operatorname{tr}(\mathbf{X})$ to signify the trace of the square matrix $\mathbf{X}, \operatorname{etr}(\mathbf{X})$ to denote $\exp (\operatorname{tr} \mathbf{X}), \mathbf{X}^{\dagger}$ to mean the Hermitian transposition. The symbol $\otimes$ denotes the Kronecker product of two matrices, ' $\sim$ ' means 'distributed as', $C W_{p}(n, \Sigma)$ is a complex Wishart distribution, $C N(\mu, \Sigma)$ is a complex vector variate Gaussian distribution, $C N_{p, q}(\mathbf{M}, \mathbf{A} \otimes \mathbf{B})$ means a complex $p \times q$ matrix variate Gaussian distribution and $\mathcal{E}_{\mathbf{X}}[\cdot]$ denotes expected value with respect to $\mathbf{X}$.

\section{Definitions and properties for random functions of complex matrix arguments}

\subsection{Zonal polynomials}

Zonal polynomials were introduced by James [James (1964)], and have become an essential tool for studying and expressing some useful special functions of matrix arguments (such as Hypergeometric functions of matrix arguments we will discuss).Using these special functions in matrix arguments, many distributions of quadratic forms can be obtained in a very compact form.

For $k>0$, we denote $\kappa=\left(k_{1}, k_{2}, \ldots, k_{p}\right)$ such that $k=\sum_{j=1}^{p} k_{j}, k_{1} \geq k_{2} \geq \ldots \geq k_{p} \geq 0$. Then we call $\kappa$ a partition of $k$ into $p$ parts. Partitions may be ordered lexicographically as follows. If $\kappa=\left(k_{1}, k_{2}, \ldots\right)$ and $\lambda=\left(l_{1}, l_{2}, \ldots\right)$, then we say $\kappa>\lambda$ if $k_{i}>l_{i}$ for the first index $i$ where the partitions differ. Now let $y_{1}, \ldots, y_{p}$ be $p$ variables. Then we say that the monomial $y_{1}^{k_{1}} \cdots y_{p}^{k_{p}}$ is of order $\kappa$ and that $y_{1}^{k_{1}} \cdots y_{p}^{k_{p}}$ is of higher order than $y_{1}^{l_{1}} \cdots y_{p}^{l_{p}}$ if $\kappa>\lambda$. The degree of a monomial in $p$ variables is the sum of degrees of the individual variables. The degree of a polynomial is the maximum degree of the monomials making up the polynomial. We denote by $\mathbf{V}_{k}$ the vector space of symmetric homogenous polynomials of degree $k$ in $p$ variables. Further let $\mathbf{V}_{\kappa}$ be the subspace of $\mathbf{V}_{k}$ defined by polynomials of order $\kappa$. Then $\mathbf{V}_{k}$ is the direct sum of the irreducible invariant subspaces $\mathbf{V}_{\mathcal{K}}$.

Defintion 1. Let $\mathbf{V}_{k}$ be defined on the eigenvalues of a $p \times p$ Hermitian matrix $\mathbf{X}$. Then the polynomial $(\operatorname{tr} \mathbf{X})^{k} \in \mathbf{V}_{k}$ has a unique decomposition into polynomials $C_{\kappa}(\mathbf{X}) \in \mathbf{V}_{\kappa}$ according to

$$
(\operatorname{tr} \mathbf{X})^{k}=\sum_{k} C_{\kappa}(\mathbf{X})
$$

The component of $(\operatorname{tr} \mathbf{X})^{k}$ in $\mathbf{V}_{\kappa}, C_{\kappa}(\mathbf{X})$, is called a zonal polynomial of $\mathbf{X}$.

The zonal polynomial $C_{\kappa}(\mathbf{X})$ is defined for all $k$ and $p$, but for a partition $\kappa$ of $k$ into more than $p$ parts, it is identically zero. The zonal polynomials have the following useful properties.

Property 1. For a scalar a,

$$
C_{\mathcal{K}}(a \mathbf{X})=a^{k} C_{\mathcal{K}}(\mathbf{X}) .
$$


Let $\kappa=\left(k_{1}, k_{2}, \ldots, k_{p}\right)$ be a partition of $k$. We will denote the complex multivariate hypergeometric coefficient by

$$
[a]_{\kappa}=\prod_{i=1}^{p}(a-i+1)_{k_{i}}
$$

where $(x)_{n}=x(x+1) \cdots(x+n-1)$ is the Pochhammer symbol [James (1964)]. Note that $(x)_{0}=1$.

Property 2. For $p$ and $q$,

$$
\frac{C_{\kappa}\left(\mathbf{I}_{p}\right)}{C_{\kappa}\left(\mathbf{I}_{q}\right)}=\frac{[p]_{\kappa}}{[q]_{\kappa}}
$$

Property 3.

$$
C_{\kappa}(\mathbf{X})=C_{\kappa}\left(\mathbf{U X U}^{\dagger}\right)
$$

where $\mathbf{U} \in \mathbf{U}(p)$, and $\mathbf{U}(p)$ is the group of all $p \times p$ complex unitary matrices.

Property 4.

$$
\int_{\mathbf{U}(p)} C_{\kappa}\left(\mathbf{U}^{\dagger} \mathbf{X U Y}\right)[d \mathbf{U}]=C_{\kappa}(\mathbf{X}) C_{\kappa}(\mathbf{Y}) / C_{\kappa}\left(\mathbf{I}_{p}\right)
$$

where $[d \mathbf{U}]$ is the invariant measure on the unitary group $\mathbf{U}(p)$ normalized to make the total measure unity.

A general formula for the coefficients of zonal polynomials has not been found. For more detail study of zonal polynomials, the reader is referred to [Muirhead (1982)].

\subsection{Hypergeometric functions of matrix arguments}

Many matrix variate distributions, especially central quadratic form distributions, can be written in terms in hypergeometric functions of matrix argument [James (1964)]-[Khatri (1965)]. Hypergeometric functions of matrix argument is a natural generalization of (generalized) hypergeometric functions of scalar argument, which have been used widely in the field of science and engineering.

Defintion 2. Let $\mathbf{X}$ be a $p \times p$ Hermitian matrix. Then hypergeometric functions of one complex matrix argument is defined by

$$
{ }_{m} \tilde{F}_{n}^{(p)}\left(a_{1}, \ldots, a_{m} ; b_{1}, \ldots, b_{n} ; \mathbf{X}\right)=\sum_{k=0}^{\infty} \sum_{\kappa} \frac{\left[a_{1}\right]_{\kappa} \ldots\left[a_{m}\right]_{\kappa} C_{\kappa}(\mathbf{X})}{\left[b_{1}\right]_{\kappa} \ldots\left[b_{n}\right]_{\kappa} k !}
$$

where $a_{1}, \ldots, a_{m} ; b_{1}, \ldots, b_{n}$ are arbitrary complex numbers, $\sum_{\kappa}$ denotes summation over all partition $\kappa$.

For the conditions for convergence of the mentioned-above series, the reader is referred to [Gupta \& Nagar (2000)]. From Definition 2 it follows that

$$
{ }_{0} \tilde{F}_{0}^{(p)}(\mathbf{X})=\operatorname{etr}(\mathbf{X})
$$

and

$$
{ }_{1} \tilde{F}_{0}^{(p)}(a ; \mathbf{X})=|\mathbf{I}-\mathbf{X}|^{-a}
$$


Defintion 3. Let $q \leq p$. Then the hypergeometric functions of two Hermitian matrices $\mathbf{X}(p \times p)$ and $\mathbf{Y}(q \times q)$ is defined by

$$
{ }_{m} \tilde{F}_{n}^{(p, q)}\left(a_{1}, \ldots, a_{m} ; b_{1}, \ldots, b_{n} ; \mathbf{X}, \mathbf{Y}\right)=\sum_{k=0}^{\infty} \sum_{\kappa} \frac{\left[a_{1}\right]_{\kappa} \ldots\left[a_{m}\right]_{\kappa} C_{\kappa}(\mathbf{X}) C_{\kappa}(\mathbf{Y})}{\left[b_{1}\right]_{\kappa} \ldots\left[b_{n}\right]_{\kappa} C_{\kappa}\left(\mathbf{I}_{p}\right) k !}
$$

The hypergeometric functions of two Hermitian matrices have the following properties.

\section{Property 5.}

$$
\int_{\mathbf{U}(p)}{ }_{m} \tilde{F}_{n}^{(p, p)}\left(a_{1}, \ldots, a_{m} ; b_{1}, \ldots, b_{n} ; \mathbf{X U Y U} \mathbf{U}^{\dagger}\right)[d \mathbf{U}]={ }_{m} \tilde{F}_{n}^{(p, p)}\left(a_{1}, \ldots, a_{m} ; b_{1}, \ldots, b_{n} ; \mathbf{X}, \mathbf{Y}\right) .
$$

Property 6.

$$
{ }_{m} \tilde{F}_{n}^{(p, p)}\left(a_{1}, \ldots, a_{m} ; b_{1}, \ldots, b_{n} ; \mathbf{I}_{p}, \mathbf{X}\right)={ }_{m} \tilde{F}_{n}^{(p)}\left(a_{1}, \ldots, a_{m} ; b_{1}, \ldots, b_{n} ; \mathbf{X}\right) .
$$

For more detail study of hypergeometric functions of matrix arguments, the reader is referred to [Gupta \& Nagar (2000)]. The hypergeometric functions of two Hermitian matrices can be expressed in terms of scalar hypergeometric functions [Khatri (1966)], which is the practical relevance of our some results given in follow-up parts.

\subsection{Generalized Hermitian polynomials of matrix arguments}

Hayakawa in 1969 gave the definition of generalized Hermite polynomial of real matrix argument $H_{\mathcal{K}}(\mathbf{T})$, and extended the definition to the case of two real matrix arguments: $P_{\mathcal{K}}(\mathbf{T}, \mathbf{A})$. Crowther in 1975 further extended it to the case of three real matrix arguments : $P_{\mathcal{K}}(\mathbf{T}, \mathbf{A}, \mathbf{B})$. Now we introduce the definition of generalized Hermite polynomial of complex matrix argument and its extensions. These functions of matrix arguments play an important role in the study of the distribution of some quadratic forms.

Defintion 4. Let $\mathbf{T}: p \times q$ and $\mathbf{W}: p \times q$, be arbitrary complex matrices, then the generalized Hermite polynomial with a complex matrix argument $H_{\mathcal{K}}(\mathbf{T})$ which corresponds to the partition $\kappa=$ $\left(k_{1}, k_{2}, \ldots, k_{p}\right)$ of $k$ is defined as:

$$
H_{\mathcal{K}}(\mathbf{T})=\pi^{-p q} e \operatorname{tr}\left(\mathbf{T T}^{\dagger}\right) \int_{\mathbf{W}} e \operatorname{tr}\left[-\mathbf{W} \mathbf{W}^{\dagger}-\imath\left(\mathbf{T W}^{\dagger}+\mathbf{W} \mathbf{T}^{\dagger}\right)\right] C_{\kappa}\left(-\mathbf{W} \mathbf{W}^{\dagger}\right) d \mathbf{W}
$$

where $\imath=\sqrt{-1}$.

It should be noted that (13) can be regarded as the Fouier transform of etr $\left[-\mathbf{W} \mathbf{W}^{\dagger}\right] C_{\kappa}\left(-\mathbf{W} \mathbf{W}^{\dagger}\right)$. The distributions of the latent roots of a noncentral Wishart matrix and of related statistics can be expressed as series of generalized Hermite polynomials.

Defintion 5. Let $p \leq q$, and let $\mathbf{T}: p \times q$ and $\mathbf{W}: p \times q$, be arbitrary complex matrices, and let $\mathbf{A}: q \times q$ and $\mathbf{B}: p \times p$ be Hermitian positive definite matrices; then the generalized Hermite polynomial with three complex matrix arguments $P_{\mathcal{K}}(\mathbf{T}, \mathbf{A}, \mathbf{B})$ which corresponds to the partition $\kappa=$ $\left(k_{1}, k_{2}, \ldots, k_{p}\right)$ of $k$ is defined as:

$$
P_{\mathcal{K}}(\mathbf{T}, \mathbf{A}, \mathbf{B})=\pi^{-p q} \operatorname{etr}\left(\mathbf{T T}^{\dagger}\right) \int_{\mathbf{W}} \operatorname{etr}\left[-\mathbf{W} \mathbf{W}^{\dagger}-\imath\left(\mathbf{T W}^{\dagger}+\mathbf{W} \mathbf{T}^{\dagger}\right)\right] C_{\mathcal{K}}\left(-\mathbf{B} \mathbf{W} \mathbf{A} \mathbf{W}^{\dagger}\right) d \mathbf{W} .
$$


The generalized Hermite polynomial with three complex matrix arguments $P_{\mathcal{K}}(\mathbf{T}, \mathbf{A}, \mathbf{B})$ has several simple properties as follows.

Property 7.

$$
P_{\mathcal{K}}\left(\mathbf{T}, \mathbf{I}_{q}, \mathbf{I}_{p}\right)=P_{\kappa}\left(\mathbf{T}, \mathbf{I}_{q}\right)=H_{\mathcal{K}}(\mathbf{T}) .
$$

Property 8.

$$
P_{\mathcal{K}}\left(\mathbf{T}, \mathbf{A}, \mathbf{I}_{p}\right)=P_{\mathcal{K}}(\mathbf{T}, \mathbf{A}) .
$$

Property 9.

$$
P_{\mathcal{K}}(\mathbf{0}, \mathbf{A}, \mathbf{B})=(-1)^{k}[q]_{\mathcal{K}} \frac{C_{\kappa}(\mathbf{A}) C_{\kappa}(\mathbf{B})}{C_{\kappa}\left(I_{q}\right)} .
$$

Crowther has calculated the polynomial $P_{\kappa}(\mathbf{T}, \mathbf{A}, \mathbf{B})$ for some special $\kappa$. With general $\kappa$, however, there is no formula available for their calculation. For more detail study of Hermitian functions of matrix argument, the reader is referred to [Gupta \& Nagar (2000)] and [Mathai et al. (1995)].

\section{Ergodic capacity of MIMO systems with interference and correlation}

\subsection{System model}

We consider a wireless link equipped with $t$ antenna elements at the transmitter and $r$ antenna elements at the receiver. It is assumed that the system is interference-limited, and there are a total of $\ell$ interfering users each equipped with $t_{i}$ antenna elements, $i=1, \ldots, \ell$. The received $r \times 1$ vector at the desired user's receiver can thus be modeled as

$$
\mathbf{y}=\mathbf{H s}+\sum_{i=1}^{\ell} \mathbf{H}_{i} \mathbf{s}_{i}
$$

where $\mathbf{H}$ is the $r \times t$ normalized channel complex matrix with Gaussian distribution [Gupta \& Nagar (2000)]: $\mathbf{H} \sim C N_{r, t}(\mathbf{0}, \mathbf{\Sigma} \otimes \mathbf{\Psi}), \mathbf{\Sigma} \otimes \mathbf{\Psi}$ is the covariance matrix of random matrix $\mathbf{H} ; \mathbf{s}$ is the $t \times 1$ transmitted data vector for the desired user with covariance matrix $\mathcal{E}\left(\mathbf{s s}^{\dagger}\right)=\mathbf{R}_{s}$ and total transmitting power $\operatorname{tr}\left(\mathbf{R}_{s}\right)=E_{s}$. Similarly, for the $i$-th co-channel interferer, $\mathbf{H}_{i}$ and $\mathbf{s}_{i}$ are the $r \times t_{i}$ normalized channel matrix and the $t_{i} \times 1$ transmitted vector with short-term average power $E_{i}$ per antenna, respectively. It is assumed that $\mathbf{H}_{i} \sim C N_{r, t_{i}}\left(\mathbf{0}, \boldsymbol{\Sigma}_{i} \otimes \mathbf{\Psi}_{i}\right)$ and $\mathbf{s}_{i} \sim C N_{t_{i}}\left(\mathbf{0}, \mathbf{R}_{i}\right)$.

Now we take a closer look at the correlation structure of $\mathbf{H}$ and $\mathbf{H}_{i}$ in (18). The correlations of the matrices $\mathbf{H}$ and $\mathbf{H}_{i}$ are specified by $\boldsymbol{\Sigma} \otimes \mathbf{\Psi}$ and $\boldsymbol{\Sigma}_{i} \otimes \mathbf{\Psi}_{i}$, respectively. Physically, $\boldsymbol{\Sigma}$ and $\boldsymbol{\Sigma}_{i}$ represent the $r \times r$ correlation matrices of incoming signal and interference at the receiver, respectively. Correspondingly, the transmit-antenna correlations for the desired user is characterized by the $t \times t$ correlation matrix $\mathbf{\Psi}$, whereas its counterpart for interferer $i$ is specified by the $t_{i} \times t_{i}$ correlation matrix $\Psi_{i}$. The structure of these correlation matrices depends on channel's fading characteristics, geometry and polarization of antenna arrays, and signal/interferers angle of arrival and spread, as described in various references [Chuah et al. (2002)].

The mentioned-above correlated MIMO channel model is one of several classical correlated MIMO channel models [Kermoal et al. (2002)]. It is very convenient for mathematical 
tractability, and has been used by many papers [Kiessling (2005)], [Paulraj et al. (2003)]. With it, the MIMO channel correlation is separable [Kermoal et al. (2002)], [Paulraj et al. (2003)], i.e.,

$$
\mathbf{H} \simeq \mathbf{A}^{\dagger} \mathbf{H}_{w} \mathbf{B}
$$

where

$$
\begin{aligned}
& \boldsymbol{\Sigma}=\mathbf{A}^{\dagger} \mathbf{A} \\
& \mathbf{\Psi}=\mathbf{B}^{\dagger} \mathbf{B}
\end{aligned}
$$

and $\mathbf{H}_{w} \sim C N_{r, t}\left(\mathbf{0}, \mathbf{I}_{r} \otimes \mathbf{I}_{t}\right)$ is a $r \times t$ random matrix of i.i.d Gaussian elements. For simplicity, just as in [Blum et al. (2002)], [Kang et al. (2003)] and [Kang et al. (2007)], all of the interfering signals $\mathbf{s}_{i}, i=1, \ldots, \ell$, are assumed to be not known at the desired user's receiver, and they are all modeled as complex Gaussian vectors. Hence, the interference $\sum_{i=1}^{\ell} \mathbf{H}_{i} \mathbf{s}_{i}$ conditioned on $\mathbf{H}_{i}, i=1, \ldots, \ell$, is complex Gaussian with covariance matrix

$$
\begin{aligned}
\mathbf{R}_{c} & =\sum_{i=1}^{\ell} \mathbf{H}_{i} \mathbf{R}_{i} \mathbf{H}_{i}^{\dagger} \\
& =\mathbf{H}_{I} \mathbf{R}_{I} \mathbf{H}_{I}^{\dagger}
\end{aligned}
$$

where

$$
\mathbf{H}_{I}=\left(\mathbf{H}_{1}, \cdots, \mathbf{H}_{\ell}\right)
$$

and

$$
\mathbf{R}_{I}=\operatorname{diag}\left(\mathbf{R}_{1}, \cdots, \mathbf{R}_{\ell}\right) .
$$

This implies that the interference is whitened by multiplying $\mathbf{y}$ by $\mathbf{R}_{c}^{-1 / 2}$.

For analytical tractability, it is assumed firstly that $\Sigma_{1}=\Sigma_{2}=\cdots=\Sigma_{\ell}=\Sigma_{I}$. Note that our assumption is more general than in the literature [Catreux et al. (2000)]-[Kang \& Alouini (2003a)] where all correlation receive matrices for interferers are identity ones, namely $\Sigma_{1}=\Sigma_{2}=\cdots=\Sigma_{\ell}=\mathrm{I}$. In order to obtain easy-to-compute closed-form expressions which provide useful insight, we have to assume further that $E_{1}=E_{2}=\cdots=E_{\ell}=E_{I}$, $\mathbf{\Psi}=\mathbf{I}_{t}$ and $\Psi_{1}=\mathbf{I}_{t_{1}}, \boldsymbol{\Psi}_{2}=\mathbf{I}_{t_{2}}, \cdots, \Psi_{\ell}=\mathbf{I}_{t_{\ell}}$. These assumptions are valid when the interfering signals come from approximately same distance from the receiver and the shadowing effects are small. However, it will lead to a pessimistic estimate of system performance if the total interfering power is fixed [Ye \& Blum (2005)]. Exactly, under these assumptions, what we will finally obtain is indeed a lower bound on the ergodic capacity for the general case. To make the problem mathematically tractable, these assumptions are usually adopted for the performance analysis of MIMO systems [Kang \& Alouini (2003a)], [Kang \& Alouini (2004b)], and [Zhang \& Cui (2004)]. Moreover, we assume that perfect channel information is available to the receiver, but the transmitter has no channel information. Then the optimum $\mathbf{R}_{S}$ to maximize the instantaneous capacity is given by $\mathbf{R}_{s}=\frac{E_{s}}{t} \mathbf{I}_{t}$. For that, we can assume that $\mathbf{R}_{s}=\frac{E_{s}}{t} \mathbf{I}_{t}$, and $\mathbf{R}_{1}=E_{1} \mathbf{I}_{t_{1}}, \mathbf{R}_{2}=E_{2} \mathbf{I}_{t_{2}}, \cdots, \mathbf{R}_{\ell}=E_{\ell} \mathbf{I}_{t_{\ell}}$.

Under all these assumptions made above, we will derive some statistical expressions only with respect to the random matrix $\rho \mathbf{H}^{\dagger}\left(\mathbf{H}_{I} \mathbf{H}_{I}^{\dagger}\right)^{-1} \mathbf{H}$, where $\rho=\frac{E_{s}}{t E_{I}}$. It should be noticed that from Chapter 3 of [Gupta \& Nagar (2000)] for the general settings of $\left\{E_{i}\right\}$ and $\left\{\Sigma_{i}\right\}$ we can approximate with high precision $\mathbf{R}_{c}$ by only using a single Wishart-distributed matrix (e.g., $\mathbf{H}_{1} \mathbf{H}_{1}^{\dagger}$ ), and thus (18) can be still used as a good approximating model for the general case. 


\subsection{Moment-generating function of mutual information}

The instantaneous mutual information $I(\mathbf{s}, \mathbf{y})$ between input vector $\mathbf{s}$ and output $\mathbf{y}$ of the MIMO link according to (18) is given by Blum et al. (2002)

$$
I(\mathbf{s}, \mathbf{y})=\log _{2}\left|\mathbf{I}_{t}+\mathbf{Q}\right|,
$$

where

$$
\mathbf{Q}=\mathbf{R}_{s} \mathbf{H}^{\dagger} \mathbf{R}_{c}^{-1} \mathbf{H}
$$

Now let $\tilde{\mathbf{H}} \sim C N_{r, t}\left(\mathbf{0}, \tilde{\boldsymbol{\Sigma}} \otimes \mathbf{I}_{t}\right)$ with

$$
\tilde{\Sigma}=\mathbf{A}^{\dagger} \Sigma_{I}^{-1} \mathbf{A}
$$

here $\mathbf{A}$ is defined in (20), and let $\tilde{\mathbf{H}}_{I} \sim C N_{r, t_{I}}\left(\mathbf{0}, \mathbf{I}_{r} \otimes \mathbf{I}_{t_{I}}\right)$ with

$$
t_{I}=\sum_{i=1}^{\ell} t_{i} .
$$

From the proof of Theorem 7.4.1 of [Gupta \& Nagar (2000)], it can follow that $\mathbf{Q}$ can be reexpressed as

$$
\mathbf{Q}=\rho \tilde{\mathbf{H}}^{\dagger}\left(\tilde{\mathbf{H}}_{I} \tilde{\mathbf{H}}_{I}^{\dagger}\right)^{-1} \tilde{\mathbf{H}}
$$

The MGF of mutual information $I(\mathbf{s}, \mathbf{y})$ is defined as

$$
M(\theta)=\mathcal{E}_{\mathbf{Q}}\left[\exp \left(\theta I(\mathbf{s}, \mathbf{y}) / \log _{2} e\right)\right]=\mathcal{E}_{\mathbf{Q}}\left|\mathbf{I}_{t}+\mathbf{Q}\right|^{\theta}
$$

Furthermore, the MGF $M(\theta)$ can be written in terms of hypergeometric functions of one matrix argument over complex field ${ }_{2} \tilde{F}_{1}^{(r)}$.

Theorem 1. Suppose that the number of receive antennas for the desired user is equal to or less than the total number of transmit antenna for the interferers, namely $r \leq t_{I}$. Then we have that

$$
M(\theta)=\frac{\tilde{\Gamma}_{r}\left(t+t_{I}\right) \tilde{\Gamma}_{r}\left(t_{I}-\theta\right)}{\tilde{\Gamma}_{r}\left(t_{I}\right) \tilde{\Gamma}_{r}\left(t+t_{I}-\theta\right)} 2 \tilde{F}_{1}^{(r)}\left(-\theta, t ; t+t_{I}-\theta ; \mathbf{I}-\rho \tilde{\boldsymbol{\Sigma}}\right) ;
$$

where $\tilde{\Gamma}_{r}(\cdot)$ is the complex multivariate gamma function defined by

$$
\tilde{\Gamma}_{r}(m)=\pi^{r(r-1) / 2} \prod_{i=1}^{r} \Gamma(m-i+1) .
$$

The proof of Theorem 1 is placed in 6.1.

It should be pointed out that in order to make the problem mathematically tractable, the assumption of $r \leq t_{I}$ is usually adopted for the performance analysis of MIMO systems [Kang \& Alouini (2003a)], [Kang \& Alouini (2004b)], and [Zhang \& Cui (2004)]. In downlink transmission, this particularly true for small and lightweight hand-held/portable receive terminals for which the size of practical adaptive array will typically be restricted to one or two antenna elements.

A general hypergeometric function of one Hermitian matrix argument can be expressed in terms of scalar hypergeometric functions [Kiessling (2005)], which is of the practical relevance of Theorem 1. 
The Vandermonde matrix with respect to a $p \times p$ diagonal matrix $\Lambda=\operatorname{diag}\left(\lambda_{1}, \lambda_{2}, \cdots, \lambda_{p}\right)$ with $\lambda_{1}>\lambda_{2}>\cdots>\lambda_{p}$ can be denoted by

$$
V(\boldsymbol{\Lambda})=\left(\lambda_{i}^{p-j}\right)
$$

Lemma 1. Let $\mathbf{W}$ be a $p \times p$ Hermitian matrix. Define $\boldsymbol{\Theta}=\operatorname{eig}(\mathbf{W})=\operatorname{diag}\left(\omega_{1}, \ldots, \omega_{p}\right)$ with $\omega_{1}>\ldots>\omega_{p}$. Then

$$
{ }_{m} \tilde{F}_{n}^{(p)}\left(a_{1}, \ldots, a_{m} ; b_{1}, \ldots, b_{n} ; \mathbf{W}\right)=\frac{|\mathbf{F}|}{|V(\boldsymbol{\Theta})|}
$$

where $\mathbf{F}=\left[f_{i j}\right]$ with

$$
f_{i j}=\omega_{i}^{p-j}{ }_{m} F_{n}\left(a_{1}-j+1, \ldots, a_{m}-j+1 ; b_{1}-j+1, \ldots, b_{n}-j+1 ; \omega_{i}\right)
$$

for $i, j=1,2, \ldots, p$.

Remark 1. The scalar hypergeometric functions appearing in the above lemma are built-in functions in computational software packages such as Mathematica.

Remark 2. When some of the $\omega_{j}$ 's are equal, we obtain the results as limiting case on the right of (34) via L'Hospital's rule.

\subsection{Ergodic capacity of MIMO systems}

A MGF uniquely defines a probability distribution. Once we can find the MGF of mutual information, we can determine all moments, including the practically important first moment, which is also known as ergodic capacity in MIMO literature[Catreux et al. (2000)]-[Kang et al. (2007)]:

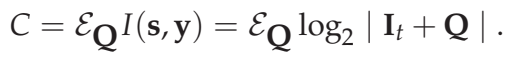

The standard approach to determine the ergodic capacity is just to find first the related MGF. Specially,

$$
C=\left.\log _{2} e \cdot \frac{\partial M(\theta)}{\partial \theta}\right|_{\theta=0} .
$$

Furthermore, After a lengthy process (see 6.2 for details), we can obtain the following expression of the ergodic capacity of MIMO systems in terms of scalar hypergeometric functions.

Theorem 2. Suppose that $r \leq t_{I}$. Let $\boldsymbol{\Delta}=\operatorname{eig}\left(\mathbf{I}_{r}-\rho \tilde{\mathbf{\Sigma}}\right)=\operatorname{diag}\left(\delta_{1}, \delta_{2}, \cdots, \delta_{r}\right)$ with $\delta_{1}>\delta_{2}>$ $\cdots>\delta_{r}$.

a) When $r \leq t$, then

$$
C=\sum_{k=1}^{r} \sum_{j=0}^{t-1} \frac{\log _{2} e}{t_{I}-k+1+j}+\frac{\log _{2} e \cdot \sum_{h=1}^{r}|\mathbf{D}(h)|}{|V(\Delta)|}
$$

where $D(h)=\left(d_{i, j}(h)\right)$ is an $r \times r$ matrix satisfying

$$
d_{i, j}(h)= \begin{cases}\sum_{k=0}^{j-1} \frac{(-j+1)_{k}(t-j+1)_{k} \delta_{i}^{r-j+k}}{\left(t+t_{I}-j+1\right)_{k} k !}, & j \neq h \\ h_{i, j}-\left(\sum_{b=0}^{t-j} \frac{1}{t_{I}+b}\right) \sum_{k=0}^{j-1} \frac{(-j+1)_{k}(t-j+1)_{k} \delta_{i}^{r-j+k}}{\left(t+t_{I}-j+1\right)_{k} k !}, j=h\end{cases}
$$


b) When $r>t$, then

$$
C=\sum_{k=1}^{r} \sum_{j=0}^{t-1} \frac{\log _{2} e}{t_{I}-k+1+j}+\frac{\log _{2} e \cdot \sum_{h=1}^{t}|\mathbf{D}(h)|}{|V(\Delta)|}
$$

where $D(h)=\left(d_{i, j}(h)\right)$ is an $r \times r$ matrix satisfying

$$
d_{i, j}(h)= \begin{cases}\sum_{k=0}^{j-1} \frac{(-j+1)_{k}(t-j+1)_{k} \delta_{i}^{r-j+k}}{\left(t+t_{I}-j+1\right)_{k} k !}, & j \neq h, j \leq t \\ \delta_{i}^{r-j}, & j \neq h, j>t \\ h_{i, j}-\left(\sum_{b=0}^{t-j} \frac{1}{t_{I}+b}\right) \sum_{k=0}^{j-1} \frac{(-j+1)_{k}(t-j+1)_{k} \delta_{i}^{r-j+k}}{\left(t+t_{I}-j+1\right)_{k} k !}, j=h .\end{cases}
$$

Here

$$
h_{i, j}=\delta_{i}^{r-j} \frac{\Gamma\left(t+t_{I}-j+1\right)}{\Gamma\left(t_{I}\right) \Gamma(t-j+1)} \int_{0}^{1} x^{t-j}(1-x)^{t_{I}-1}\left(1-\delta_{i} x\right)^{j-1}\left[\ln \left(1-\delta_{i} x\right)-\ln (1-x)\right] d x .
$$

\subsection{Numerical examples and remarks}

Now we offer some numerical examples validating the analysis and showing the effect of various system parameters on the ergodic capacity of MIMO systems. For simplicity, we adopt the correlation model of exponential type (see [Loyka (2001)] and [Kiessling (2005)]) at the receiver with

$$
\begin{gathered}
\Sigma=\left[\beta^{|i-j|}\right] \\
\Sigma_{I}=\left[\beta_{I}^{|i-j|}\right]
\end{gathered}
$$

The correlation coefficients $\beta$ and $\beta_{I}$ are for the desired user and interferers, respectively. They range from 0 to 1 . Here 0 means that the correlation is the weakest, and 1 means that the correlation is the strongest. Furthermore, the SIR in dB is defined by $10 \log _{10}\left(\frac{E_{s}}{t_{I} E_{I}}\right)$ which characterizes the signal to interference ratio in the considered physical condition.

The ergodic capacity versus the SIR is depicted in Fig.1 where the four curves are shown for four different correlation coefficients equal to $\beta=0.3,0.6,0.8,0.9$, respectively. The considered MIMO system possesses 4 transmit antennas and 4 receive antennas with 10 interfering antennas. The correlation coefficient $\beta_{I}$ is set at 0.4 . As expected, the ergodic capacity decreases with increasing $\beta$. It can be further seen that the effect of strong correction on the capacity is significant.

Fig.2 depicts the ergodic capacity versus the SIR for four different correlations. The four curves in Fig. 2 are shown for interfering correlation coefficients equal to $\beta_{I}=0.3,0.6,0.8,0.9$, respectively. The considered MIMO system is with 2 transmit antennas and 4 receive antennas and interfered by a user with 8 antennas. The correlation coefficient is set at $\beta=0.5$. It can be seen from Fig. 2 that the impact of correlation for interferers on the ergodic capacity increases with increased interfering correlation coefficient $\beta_{I}$. Therefore, the interference correlation is beneficial, especially the strong correlation.

Simulation results are included in Figs.1-2 for comparison. Each point in the simulation curves are obtained by averaging over 100,000 independent computer runs. The theoretical and simulation results are nearly identical verifying the validity of the theory. Consequently, in the following evaluations, we only consider the theoretical results. 


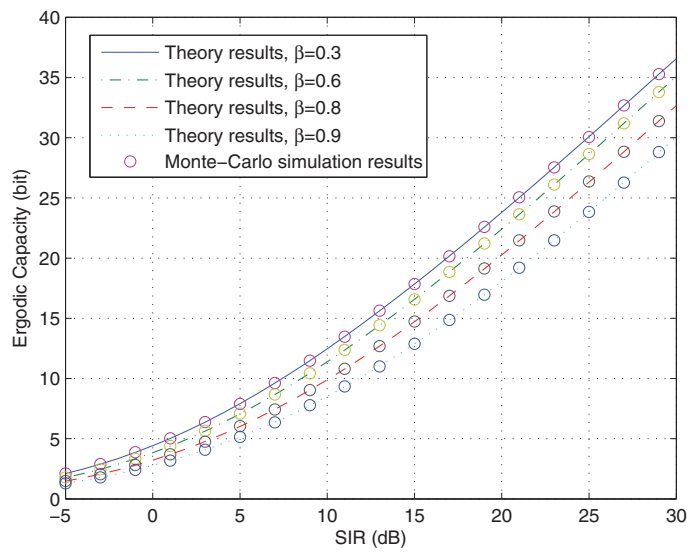

Fig. 1. Ergodic capacity versus SIR for different signal channel correlations.

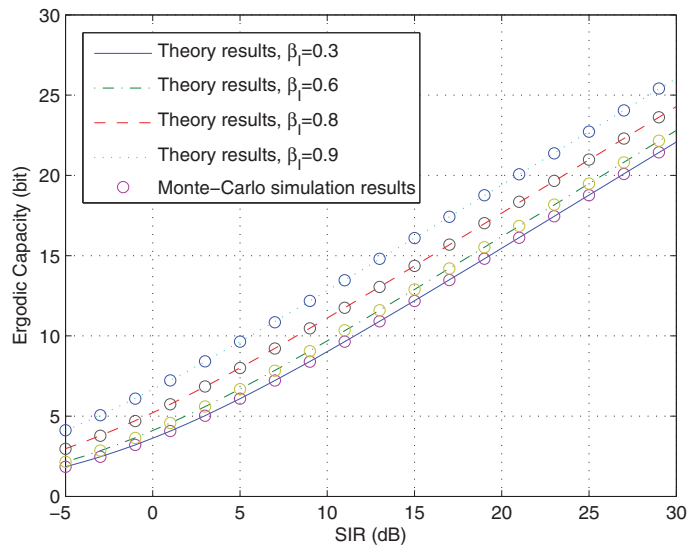

Fig. 2. Ergodic capacity versus SIR for different interfering correlations.

In Fig.3, a MIMO system with 4 transit antennas and 4 receive antennas is considered. We assume only 1 interferer is involved in this system. We observe the ergodic capacities with various interference antennas. In Fig.3, the four curves correspond to the number of total interfering transmit antennas $t_{I}=4,5,6,7$, respectively. It can be observed that the ergodic capacity drops as $t_{I}$ increases, and the drop becomes gradually slow.

Finally, in Fig.4, we compare our analytical results (neglecting the noise component) with the Monte-Carlo simulation results with Gaussian noise involved in the corresponding physical conditions. We set the transmit power in the interest system at $30 \mathrm{~dB}$, and let $\beta$ and $\beta_{I}$ be qual to 0.4 and 0.8 , respectively. Furthermore, we assume the system is interfered by a user with 10 antennas. We plot the curves with $t=r=2,3$ and 4, respectively. As shown in the figure, our 


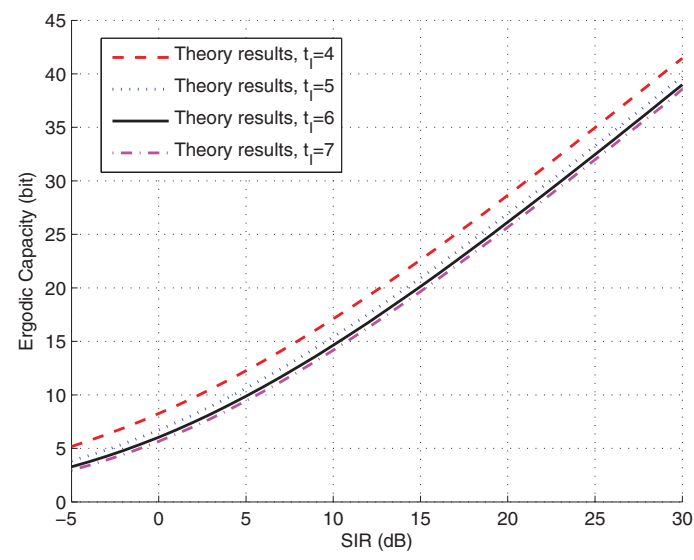

Fig. 3. Ergodic capacity versus SIR for various interfering antenna configurations.

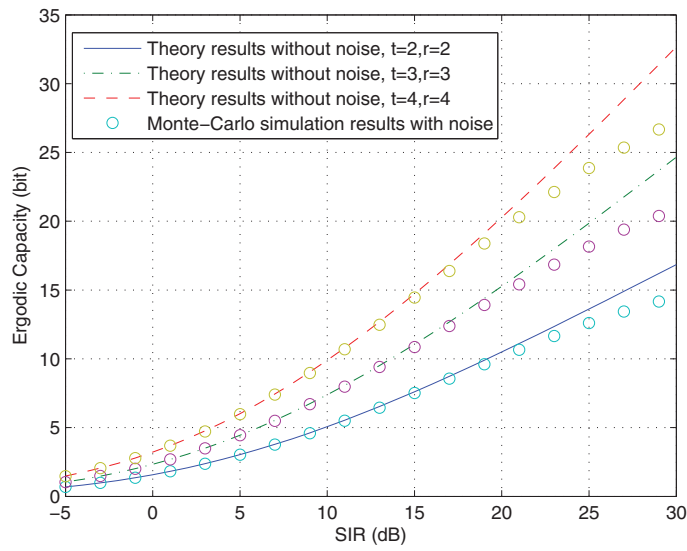

Fig. 4. Ergodic capacity versus SIR for various antenna configurations.

analytical results match the simulation results under low SIRs, however, we lose the precision gradually as SIR grows.

\section{Outage performance of TRD MIMO systems with interference and correlation}

\subsection{System model}

Suppose the intended user employs $r$ antennas to receive signals transmitted from $t$ antennas. The channels that link the $t$ transmit and $r$ receive antennas are characterized by an $r \times t$ matrix $\mathbf{H}$, which is assumed to follow the joint complex Gaussian distribution with mean matrix $\mathbf{M}$ 
and covariance matrix $\Sigma \otimes \Psi$. Symbolically, we will write

$$
\mathbf{H} \sim C N_{r, t}(\mathbf{M}, \mathbf{\Sigma} \otimes \mathbf{\Psi})
$$

where $\Psi$ and $\Sigma$ define the correlation structure at the transmit and receive ends, respectively. It is assumed that the intended signal is corrupted by $\ell$ independent interferers, and the $i$ th interferer transmits its signal with $t_{i}$ antennas where $i=1, \ldots, \ell$. The desired information symbol $b_{0}$ is weighted by the transmit beamformer $\mathbf{u}$ before being feeded to the $t$ transmit antennas. The transmit beamformer is normalized to have a unit norm $\mathbf{u}^{\dagger} \mathbf{u}=1$ so that the transmit energy equals $E_{s}=\left|b_{0}\right|^{2}$. The $r \times 1$ vector at the desired user's receiver can thus be written as

$$
\mathbf{y}=b_{0} \mathbf{H u}+\sum_{i=1}^{\ell} \mathbf{H}_{i} \mathbf{s}_{i}+\mathbf{n},
$$

where $\mathbf{H}_{i}$ is the $r \times t_{i}$ the channel matrix characterizing the links from the desired user's $r$ receive antennas to the $t_{i}$ transmit antennas of interferer $i$; and $\mathbf{s}_{i}$ is the symbols transmitted by interferer $i$, such that $\mathcal{E}\left[\mathbf{s}_{i} \mathbf{s}_{i}^{\dagger}\right]=E_{i} \mathbf{I}_{t_{i}}$ with $E_{i}$ denoting the average symbol energy. In the way similar to defining $\mathbf{H}$, we assume

$$
\mathbf{H}_{i} \sim C N_{r, t_{i}}\left(\mathbf{M}_{i}, \boldsymbol{\Sigma}_{i} \otimes \mathbf{\Psi}_{i}\right)
$$

We assume the additive noise vector $\mathbf{n}$ to follow the $r \times 1$ complex Gaussian distribution of mean zero and covariance matrix $\mathbf{R}_{n}$. Conditioned on $\mathbf{H}_{i}, i=1, \ldots, \ell$, the covariance matrix of interference-plus-noise component is given by

$$
\mathbf{R}_{c}=\sum_{i=1}^{\ell} E_{i} \mathbf{H}_{i} \mathbf{H}_{i}^{\dagger}+\mathbf{R}_{n} .
$$

\subsection{Formulation}

The TRD system transmits one symbol at a time, and employs a weighting vector $\mathbf{w}$ to combine received vector $\mathbf{y}$ to form a single decision variable. The transmit and receive weighting vectors, $\mathbf{u}$ and $\mathbf{w}$ should be chosen to maximize the output signal to interference-plus-noise ratio (SINR) at every time instant, as defined by

$$
\gamma=\frac{\mathbf{w}^{\dagger}(\mathbf{H u})(\mathbf{H u})^{\dagger} \mathbf{w}}{\mathbf{w}^{\dagger} \mathcal{E}_{n}\left[\left(\sum_{i=1}^{\ell} \mathbf{H}_{i} \mathbf{s}_{i}+\mathbf{n}\right)\left(\sum_{i=1}^{\ell} \mathbf{H}_{i} \mathbf{s}_{i}+\mathbf{n}\right)^{\dagger}\right] \mathbf{w}}
$$

where $\mathcal{E}_{n}$ denotes the expectation with respect to $\mathbf{n}$. The result of expectation equals $\mathbf{R}_{c}$ given in (48). Optimization of $\gamma$ is the problem of Rayleigh quotient. Given the channel-state information and conditional on $\mathbf{u}$, we optimize $\gamma$ with respect to $\mathbf{w}$ to obtain [Kang \& Alouini (2004b)]

$$
\gamma(\mathbf{u})=\frac{\mathbf{u}^{\dagger}\left(E_{S} \mathbf{H}^{\dagger} \mathbf{R}_{c}^{-1} \mathbf{H}\right) \mathbf{u}}{\mathbf{u}^{\dagger} \mathbf{u}}
$$

where we have used the fact that $\mathbf{u}^{\dagger} \mathbf{u}=1$ to represent the second line in the form of Rayleigh quotient. Thus, we can upper bound $\gamma(\mathbf{u})$ by

$$
\gamma_{\max }=\lambda_{(1)}
$$


where $\lambda_{(1)} \geq \lambda_{(2)} \geq \cdots \lambda_{(q)}$ are the non-zero eigenvalues of the matrix product

$$
\mathbf{F}=E_{s} \mathbf{H}^{\dagger} \mathbf{R}_{c}^{-1} \mathbf{H}
$$

in the descending order, and $\mathbf{v}_{(1)}, \mathbf{v}_{(2)}, \cdots, \mathbf{v}_{(q)}$ are their corresponding eigenvectors. The non-ordered eigenvalues and eigenvectors will be denoted by $\lambda_{1}, \lambda_{2}, \cdots, \lambda_{q}$ and $\mathbf{v}_{1}, \mathbf{v}_{2}, \cdots, \mathbf{v}_{q}$, respectively.

The outage probability of TRD systems can be defined directly in terms of the instantaneous SINR $\gamma_{\max }=\lambda_{(1)}$ or by channel capacity [Kang et al. (2003)]

$$
C=\log _{2}\left(1+\lambda_{(1)}\right) \text {. }
$$

Both leads to the same expression for an outage event: $\lambda_{(1)}<\Lambda$, but with the protection ratio $\Lambda$ defined differently as shown by

$$
\Lambda= \begin{cases}\gamma_{0}, & \text { outage in terms of } \gamma \\ 2^{C_{0}}-1, & \text { outage in terms of } C .\end{cases}
$$

In either case, we can write the outage probability as

$$
P_{\text {out }}=\operatorname{Pr}\left\{\lambda_{(1)}<\Lambda\right\} .
$$

To determine the outage performance, the central issue is to determine the probability density function (PDF) of $\lambda_{(1)}$ or equivalently, its cumulative density function (CDF).

Determination of the CDF of the principal eigenvalue of a rank- $q$ non-negative definite matrix of the form $\mathbf{F}=E_{S} \mathbf{H}^{\dagger} \mathbf{R}_{c}^{-1} \mathbf{H}$ has been addressed intensively in the literature [Muirhead (1982)]. The predominant methodology, however, is to arrange the sample eigenvalues in a descending order and then to determine the PDF of the largest one. The methodology is also prevailing in the area of communications [Kang \& Alouini (2004b)]. Such methodology, however, often leads to mathematically intractability except for some simple cases. In this paper, we therefore consider the non-ordered sample eigenvalues instead. The key step is to represent the outage event $\lambda_{(1)}<\Lambda$, alternatively, by virtue of non-ordered eigenvalues. To this end, we write the sample space

$$
\left.\left\{\mathbf{F}: \lambda_{(1)}<\Lambda\right)\right\}=\left\{\mathbf{F}: \cap_{i=1}^{q}\left(\lambda_{i}<\Lambda\right)\right\} .
$$

The right-hand side is further expressible in matrix form. Hence,

$$
\left\{\mathbf{F}: \lambda_{(1)}<\Lambda\right\}=\{\mathbf{F}: \mathbf{F}<\Lambda \mathbf{I}\}
$$

where $\mathbf{F}<\Lambda \mathbf{I}$ means that $(\Lambda \mathbf{I}-\mathbf{F})$ is a positive definite matrix. The equivalence of the two expressions is obvious, in much the same way as what we do in selection combining. Let $\mathbf{V}$ denote the matrix of eigenvectors of $\mathbf{F}$. Namely, $\mathbf{V}=\left(\mathbf{v}_{1}, \cdots, \mathbf{v}_{q}, \cdots, \mathbf{v}_{t}\right)$. Hence we can write

$$
\Lambda \mathbf{I}-\mathbf{F}=\mathbf{V} \operatorname{diag}\left(\Lambda-\lambda_{1}, \cdots, \Lambda-\lambda_{q}, 0, \cdots, 0\right) \mathbf{V}^{\dagger}
$$

The positive definiteness of $(\Lambda \mathbf{I}-\mathbf{F})$ implies that all of eigenvalues $\Lambda-\lambda_{i}$ are positive, and vice versa, thus showing the correctness of (57). This equivalence was previously used in Chapter 9 of [Muirhead (1982)].

We use it here to represent the outage probability yielding

$$
P_{\text {out }}=\operatorname{Pr}\{\mathbf{F}<\Lambda \mathbf{I}\} .
$$


The matrix representation of outage event, though simple in principle, provides a novel framework to tackle the outage issue of the optimal TRD system. The key to success along this direction is to find the joint cumulative distribution function of matrix $\mathbf{F}$.

For ease of presentation, we define variables

$$
\begin{aligned}
& u=\max \{r, t\} \\
& v=\min \{r, t\}
\end{aligned}
$$

and the $v \times u$ complex matrix

$$
\mathbf{Y}=\left\{\begin{array}{l}
\boldsymbol{\Sigma}^{-1 / 2} \mathbf{M} \Psi^{-1 / 2}, \quad r<t \\
\mathbf{\Psi}^{-1 / 2} \mathbf{M}^{+} \boldsymbol{\Sigma}^{-1 / 2}, t \leq r
\end{array}\right.
$$

\subsection{Outage performance with co-channel interference}

We first proceed to operational environments with co-channel interference. For mathematical tractability, let us first simplify the interference covariance matrix given in (48). We assume that the operating environment is interference-dominated, so that the noise component is negligible. Hence, we can rewrite (48) as

$$
\mathbf{R}_{c}=\sum_{i=1}^{\ell} E_{i} \mathbf{H}_{i} \mathbf{H}_{i}^{\dagger}
$$

where $\mathbf{H}_{i} \mathbf{H}_{i}^{\dagger} \sim C W_{r}\left(t_{i}, \Sigma_{i}\right)$. For the case with $E_{1}=E_{2}=\cdots=E_{\ell}=E_{I}$ and $\boldsymbol{\Sigma}_{1}=\boldsymbol{\Sigma}_{2}=\cdots=$ $\Sigma_{\ell}=\Sigma_{I}$, it is easy to use Theorem 3.2.4 of Muirhead [Muirhead (1982)] to assert that $\mathbf{R}_{c}$, up to a factor of $E_{I}$, follows the Wishart distribution, as shown by

$$
\mathbf{R}_{c} \sim C W_{r}\left(t_{I}, \Sigma_{I}\right)
$$

where $t_{I}=\sum_{i=1}^{\ell} t_{i}$. Clearly, this is the extension of the closure property of chi-square distribution. For the general setting of $E_{i}$ 's, we can accurately approximate $\mathbf{R}_{c}$ by using a single Wishart-distributed matrix, say $\mathbf{Q}_{1}$, in much the same as what we do for a sum of chi-square variables [Pearson \& Hartley (1976)]. The resulting matrix $\mathbf{Q}_{1}$ has the following distribution

$$
\mathbf{Q}_{1} \sim C W_{r}\left(t_{1}, \Sigma_{1}\right),
$$

for which the parameters $t_{1}$ and $\Sigma_{1}$ can be determined by equating the first two moments of $\mathbf{Q}_{1}$ and $\mathbf{R}_{c}$; for details, see Chapter 3 of [Gupta \& Nagar (2000)]. From the above analysis, it follows that we can use a single a Wishart-distributed matrix, say $\mathbf{Q}_{1}$, to replace $\mathbf{R}_{c}$ to simplify the analysis. It also follows that $t_{1}$ is usually much greater than the number of antennas of the intended user. Thus, without loss of the generality, we can write the decision matrix (52) as

$$
\mathbf{F}=\left(E_{s} / E_{1}\right) \mathbf{H}^{\dagger} \mathbf{Q}_{1}^{-1} \mathbf{H}
$$

whereby, for a given power protection ratio $\Lambda$, the outage probability can be written as

$$
\begin{aligned}
P_{\text {out }}(x) & =\operatorname{Pr}\{\mathbf{F}<\Lambda \mathbf{I}\} \\
& =\operatorname{Pr}\{\mathbf{J}<x \mathbf{I}\}
\end{aligned}
$$


where $x=\Lambda E_{1} / E_{S}$ and $\mathbf{J}$ is defined in terms of random channel matrices $\mathbf{H}$ and $\mathbf{Q}_{1}$, as shown by

$$
\mathbf{J}=\mathbf{H}^{\dagger} \mathbf{Q}_{1}^{-1} \mathbf{H}
$$

We assume the signal suffers from Rician fading so that the corresponding channel matrix $\mathbf{H} \sim C N_{r, t}(\mathbf{M}, \boldsymbol{\Sigma} \otimes \mathbf{\Psi})$. Suppose that the interferer employs $t_{1}$ transmit antennas such that $r \leq t_{1}$. We also assume that the $t_{1}$ channel-gain vectors for the interferer that link each transmit antenna to the $r$ receive antennas are independent and identically distributed as $C N_{r}\left(\mathbf{0}, \Sigma_{1}\right)$. Then, we can assert that $\mathbf{Q}_{1} \sim C W_{r}\left(t_{1}, \Sigma_{1}\right)$. Under these assumptions and by introducing the following matrix notations:

$$
\Delta= \begin{cases}\Sigma^{-1} \Sigma_{1}, & t \leq r \\ \Psi^{-1}, & r<t\end{cases}
$$

and

$$
\boldsymbol{\Theta}= \begin{cases}\Sigma^{-1} \Sigma_{1}, & r<t \\ \Psi^{-1}, & t \leq r\end{cases}
$$

we can explicitly work out the outage probability defined in (67), obtaining results summarized in the following theorem. The proof of this theorem is placed in 7.1.

Theorem 3. The outage probability of the optimal TRD system with co-channel interference is given by

$$
P_{\text {out }}(x)=d \sum_{k=0}^{\infty} \frac{x^{u v+k}}{k !} \sum_{\kappa} \frac{\left[t+t_{1}\right]_{\kappa}}{[u+v]_{\kappa}} P_{\mathcal{K}}(\mathbf{Y}, \Delta, \boldsymbol{\Theta})
$$

where

$$
d=\frac{\tilde{\Gamma}_{v}\left(t+t_{1}\right) \tilde{\Gamma}_{v}(v)}{\tilde{\Gamma}_{v}\left(t+t_{1}-u\right) \tilde{\Gamma}_{v}(u+v)}|\mathbf{\Delta}|^{v}|\mathbf{\Theta}|^{u} \cdot \operatorname{etr}\left[-\mathbf{Y} \mathbf{Y}^{\dagger}\right]
$$

The above generalized Hermite polynomial $P_{\kappa}(\cdot, \cdot, \cdot)$, though difficult in numerical calculation [Gupta \& Nagar (2000)], serve as a fundamental tool in the study of the distribution of some quadratic forms. Eq.(71) is a general formula, providing a solid foundation for further study. This combination can be treated as a special Rayleigh case by setting $\mathbf{M}=\mathbf{0}$. Namely, $\mathbf{H} \sim$ $C N_{r, t}(\mathbf{0}, \mathbf{\Sigma} \otimes \mathbf{\Psi})$. With the condition, Theorem 3 leads to the following corollary.

Corollary 1. Let $\mathbf{M}=\mathbf{0}$. Then

$$
P_{\text {out }}(x)=d_{1} x^{u v}{ }_{2} \tilde{F}_{1}^{(u, v)}\left(u, t+t_{1} ; u+v ; x \boldsymbol{\Delta},-\boldsymbol{\Theta}\right)
$$

where

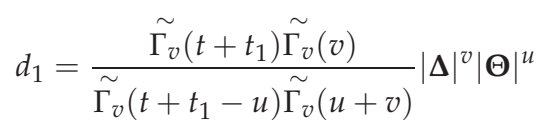

The corollary is made by inserting $\mathbf{M}=\mathbf{0}$ into (71) and invoking Property 9 in Section 2 (i.e. the complex counterpart of Expression (1.8.3) in [Gupta \& Nagar (2000)]).

Our concern is whether (72) can be further simplified. To this end, we note that when $r=t$, the hypergeometric function ${ }_{2} \tilde{F}_{1}^{(u, v)}$ involved in (72) can be converted to scalar hypergeometric functions which are much easier to calculate by using for example, the built-in functions in Matlab, Mathematica and Maple. The simplification can be done by invoking the following lemma (see Lemma 2 in [Kiessling (2005)]). 
Lemma 2. Let $\mathbf{A}=\operatorname{eig}(\mathbf{X})=\operatorname{diag}\left(\lambda_{1}, \ldots, \lambda_{p}\right)$ and $\mathbf{B}=\operatorname{eig}(\mathbf{Y})=\operatorname{diag}\left(\omega_{1}, \ldots, \omega_{p}\right)$ with $\lambda_{1}>$ $\ldots>\lambda_{p}$ and $\omega_{1}>\ldots>\omega_{p}$. Furthermore define

$$
\begin{gathered}
\Gamma_{p}(p)=\prod_{i=1}^{p} \Gamma(p-i+1), \\
\alpha_{p}(\mathbf{A})=\prod_{i<j}\left(\lambda_{i}-\lambda_{j}\right)
\end{gathered}
$$

and

$$
\Psi_{n}^{p}(\mathbf{b})=\prod_{i=1}^{p} \prod_{j=1}^{n}\left(b_{j}-i+1\right)^{i-1}
$$

for $\mathbf{b}=\left(b_{1}, b_{2}, \ldots, b_{n}\right)$. Then

$$
{ }_{m} \tilde{F}_{n}^{(p, p)}\left(a_{1}, \ldots, a_{m} ; b_{1}, \ldots, b_{n} ; \mathbf{X}, \mathbf{Y}\right)=\frac{\Gamma_{p}(p) \Psi_{n}^{p}(\mathbf{b})|\mathbf{L}|}{\alpha_{p}(\mathbf{A}) \alpha_{p}(\mathbf{B}) \Psi_{m}^{p}(\mathbf{a})}
$$

where $\mathbf{L}=\left[l_{i j}\right]$ with

$$
l_{i j}={ }_{m} F_{n}\left(a_{1}-p+1, \ldots, a_{m}-p+1 ; b_{1}-p+1, \ldots, b_{n}-p+1 ; \lambda_{i} \omega_{j}\right)
$$

for $i, j=1,2, \ldots, p$.

When some of the $\lambda_{i}{ }^{\prime}$ s or $\omega_{j}$ 's are equal, we obtain the results as limiting case on the right of (77) via L'Hospital's rule (see [Kiessling (2005)] for a detail process.)

Let us return to the general case with $r \neq t$. There is a simple method to convert this problem into the corresponding one with $r=t$. The basic skill is to obtain the exact outage probability as the result of a limiting process. The interested reader is referred to [Kiessling (2005)] for details. By the same token, we can simplify (72) to obtain an alternative expression which is much easier in numerical calculation.

Corollary 2. Let $\mathbf{D}_{\Delta}=\operatorname{eig}(\boldsymbol{\Delta})=\operatorname{diag}\left(\delta_{1}, \ldots, \delta_{u}\right)$ and $\mathbf{D}_{\Theta}=\operatorname{eig}(\boldsymbol{\Theta})=\operatorname{diag}\left(\theta_{1}, \ldots, \theta_{v}\right)$ with $\delta_{1}>\ldots>\delta_{u}$ and $\theta_{1}>\ldots>\theta_{v}$. Then

$$
P_{\text {out }}(x)=d_{2} x^{u v-u(u-1) / 2}|\mathbf{Z}|
$$

where $d_{2}$ is defined as follows

$$
d_{2}=\frac{(-1)^{u(u-1) / 2} \Gamma_{v}(v)\left[\Gamma\left(t+t_{1}-u+1\right)\right]^{v}|\Delta|^{v}|\boldsymbol{\Theta}|^{v}}{\Gamma_{v}\left(t+t_{1}-u\right)[\Gamma(v+1)]^{v} \alpha_{u}\left(\mathbf{D}_{\Delta}\right) \alpha_{v}\left(\mathbf{D}_{\Theta}\right)}
$$

and the entries of matrix $\mathbf{Z}=\left[z_{i j}\right]$ are given by

$$
z_{i j}= \begin{cases}{ }_{2} F_{1}\left(1, t+t_{1}-u+1 ; v+1 ;-x \theta_{i} \delta_{j}\right), & i \leq v \\ \left(x \delta_{j}\right)^{(i-v-1)}, & i>v .\end{cases}
$$

The expression in (71) is a general result. Its correctness can be examined by showing that the main result of [Kang \& Alouini (2004b)] is one of its special cases. 
Corollary 3. Let $\mathbf{M}=\mathbf{0}$ and $\mathbf{\Psi}=\mathbf{I}_{t}$. Then

$$
P_{\text {out }}(x)=\prod_{i=1}^{v} \frac{\left|\beta\left(\frac{x}{1+x}\right)\right| \cdot \Gamma\left(t+t_{1}-i+1\right)}{\Gamma\left(t+t_{1}-u-i+1\right) \Gamma(u-i+1) \Gamma(v-i+1)}
$$

where $\beta(y)$ is an $v \times v$ matrix function of the scalar $y$ with entries

$$
[\beta(y)]_{i j}=\beta_{y}\left(u-v+i+j-1, t_{1}-r+1\right) .
$$

The function $\beta_{y}(p, q)$ is called the incomplete beta function (see [Gradshteyn \& Ryzhik (1994)], Eqn.[8.391]).

This result is exactly the same as Eqn.(11) of [Kang \& Alouini (2004b)]. The proof is a little complicated, yet not important to us, and thus is omitted.

\subsection{Outage performance without co-channel interference}

When co-channel interference is absent, we can set $E_{i}=0, i=1, \ldots, \ell$ to rewrite (48) as

$$
\mathbf{R}_{c}=N_{0} \boldsymbol{\Phi}_{n}
$$

where $\boldsymbol{\Phi}_{n}$ has been normalized to signify the branch noise correlation matrix whereas $N_{0}$ denotes the noise variance at each branch. Now we need a difference treatment due to the replacement of the random matrix summation $\mathbf{R}_{c}=\sum_{i=1}^{\ell} E_{i} \mathbf{H}_{i} \mathbf{H}_{i}^{\dagger}$ with a constant matrix $N_{0} \boldsymbol{\Phi}_{n}$ in the quadratic form $\mathbf{F}$. Nevertheless, the procedure is parallel.

Given the change in covariance matrix $\mathbf{R}_{c}$, we need to modify $x$ and $\mathbf{J}$ accordingly, as shown by

$$
x=\Lambda N_{0} / E_{s}, \quad \mathbf{J}=\mathbf{H}^{\dagger} \boldsymbol{\Phi}_{n}^{-1} \mathbf{H} .
$$

Correspondingly, matrices $\Delta$ and $\Theta$ are modified to

$$
\boldsymbol{\Delta}= \begin{cases}\boldsymbol{\Sigma}^{-1} \boldsymbol{\Phi}_{n}, & t \leq r \\ \boldsymbol{\Psi}^{-1}, & r<t .\end{cases}
$$

and

$$
\boldsymbol{\Theta}= \begin{cases}\Sigma^{-1} \boldsymbol{\Phi}_{n}, & r<t \\ \mathbf{\Psi}^{-1}, & t \leq r .\end{cases}
$$

With these notations, we can write $P_{\text {out }}=\operatorname{Pr}\{\mathbf{J}<x \mathbf{I}\}$ which, after some manipulations as shown in 7.2 , leads to the following result.

Theorem 4. The outage probability of the optimal TRD system without co-channel interference is given by

$$
P_{\text {out }}(\mathbf{Q}<x \mathbf{I})=c \sum_{k=0}^{\infty} \frac{x^{u v+k}}{k !} \sum_{\kappa} \frac{P_{\kappa}(\mathbf{Y}, \boldsymbol{\Delta}, \mathbf{\Theta})}{[u+v]_{\kappa}}
$$

where

$$
c=\frac{\tilde{\Gamma}_{v}(v)}{\tilde{\Gamma}_{v}(u+v)}|\boldsymbol{\Delta}|^{v}|\boldsymbol{\Theta}|^{u} \cdot \operatorname{etr}\left[-\mathbf{Y} \mathbf{Y}^{\dagger}\right]
$$

An important case is Rayleigh faded signals for which $\mathbf{M}=\mathbf{0}$ and (87) can be simplified. 
Corollary 4. when $\mathbf{M}=\mathbf{0}$, we have that

$$
P_{\text {out }}=c_{1} x^{u v}{ }_{1} \tilde{F}_{1}^{(u, v)}(u ; u+v ; x \Delta,-\mathbf{\Theta})
$$

where

$$
c_{1}=\frac{\tilde{\Gamma}_{v}(v)}{\tilde{\Gamma}_{v}(u+v)}|\boldsymbol{\Delta}|^{v}|\Theta|^{u} .
$$

This corollary's proof is similar to that of Corollary 2 and thus is omitted.

Similar to ${ }_{2} \tilde{F}_{1}^{(u, v)}$, the hypergeometric function ${ }_{1} \tilde{F}_{1}^{(u, v)}$ involved in (89) can be also easily calculated by representing it in terms of scalar hypergeometric functions for ease of calculation. Specifically, by using the same techniques as used by Kiessling [Kiessling (2005)], we can obtain the following corollary.

Corollary 5. Let $\mathbf{D}_{\Delta}=\operatorname{eig}(\boldsymbol{\Delta})=\operatorname{diag}\left(\delta_{1}, \ldots, \delta_{u}\right)$ and $\mathbf{D}_{\Theta}=\operatorname{eig}(\boldsymbol{\Theta})=\operatorname{diag}\left(\theta_{1}, \ldots, \theta_{v}\right)$ with $\delta_{1}>\ldots>\delta_{u}$ and $\theta_{1}>\ldots>\theta_{v}$.

$$
P_{\text {out }}(x)=c_{2} x^{u v-u(u-1) / 2}|\mathbf{Y}|
$$

where $c_{2}$ is given by

$$
c_{2}=\frac{(-1)^{u(u-1) / 2} \Gamma_{v}(v)|\Delta|^{v}|\boldsymbol{\Theta}|^{v}}{[\Gamma(v+1)]^{v} \alpha_{u}\left(\mathbf{D}_{\Delta}\right) \alpha_{v}\left(\mathbf{D}_{\Theta}\right)},
$$

and the entry of the matrix $\mathbf{Y}=\left[y_{i j}\right]$ is given by

$$
y_{i j}= \begin{cases}{ }_{1} F_{1}\left(1 ; v+1 ;-x \theta_{i} \delta_{j}\right), & i \leq v ; \\ \left(x \delta_{j}\right)^{(i-v-1)}, & i>v .\end{cases}
$$

To examine the correctness of our results given in (89), let us consider the special case of independent noise and i.i.d. fading Rayleigh channels such that $\boldsymbol{\Phi}_{n}=\mathbf{I}$ and $\boldsymbol{\Psi}=\boldsymbol{\Sigma}=\mathbf{I}$. These conditions, when inserted into (89) and simplified, leads to (94) shown below.

Corollary 6. Let $\boldsymbol{\Phi}_{n}=\mathbf{I}$ and $\boldsymbol{\Psi}=\boldsymbol{\Sigma}=\mathbf{I}$. Then

$$
P_{\text {out }}=\frac{|\mathbf{A}(x)|}{\prod_{k=1}^{v} \Gamma(u-k+1) \Gamma(v-k+1)}
$$

where $\mathbf{A}(x)$ is a $v \times v$ matrix function with its $(i, j)$ th entries given by

$$
[\mathbf{A}(x)]_{i j}=\gamma(u-v+i+j-1, x)
$$

for $i, j=1,2, \ldots, v$.

This result is identical to the corresponding one in [Dighe et al. (2001)] and [Kang \& Alouini (2003b)]. If we further set $v=2$, then (94) can be rewritten as

$$
P_{\text {out }}=\frac{\gamma(u-1, x) \gamma(u+1, x)-\gamma(u, x)^{2}}{\Gamma(u) \Gamma(u-1)}
$$

which is exactly the same as the known result described in [Kang \& Alouini (2004a)]. Its proof is not difficult but not important and thus, is omitted. 


\subsection{Numerical results and remarks}

The validity of Theorem 3 and Theorem 4 has been rigorously examined by showing that they include most of existing results in the literature as special cases. In this section, we examine the correctness of Corollary 1 and Corollary 4 with numerical results. For simplicity, we assume that the spatial correlation among antennas follows the exponential model with correlation between antennas $p$ and $q$ given by $c(p, q)=g^{|p-q|} \exp (j(p-q) \pi / 12)$. Physically, $g^{|p-q|}$ denotes the correlation magnitude, and $g$ stands for the correlation coefficient.

We assume that the receiver is equipped with $r$ antennas for the reception of Rayleigh faded signals from $t$ intended transmit antennas. The received signals are corrupted by Rayleigh faded interference from $\ell$ interferers. Thus, Corollaries 2 and 5 are applicable in theoretical evaluation. Simulation results are also included for comparison. Each point in the simulated curves is produced by averaging over at least 100,000 independent computer runs. Throughout this section, we set $t=4$ and $r=2$, and assume that the correlation at the intended transmit and receive ends is characterized by $g_{t}$ and $g_{r}$, respectively.

We first investigate the case with co-channel interference. For ease of illustration, assume the presence of only one co-channel interferer (i.e., $\ell=1$ ) which employs $t_{1}$ antennas for transmission. Further assume that the correlation structure at the both sides of the $t_{1} \times r$ interfering channel matrix is the same, characterized by $g_{1}$.

Fig. 5 shows the variation of outage probability with the number of the interferer's transmit antennas. The parameter setting is: $g_{t}=0.5, g_{r}=0.9$, and $g_{1}=0.5$. The curves in the figure are for $t_{1}=2,3,4,10,14$, respectively. As expected, the outage performance becomes worse as $t_{1}$ increases, but the decrease magnitude becomes smaller and smaller. It is also observed that the simulated results coincide with their theoretical counterparts.

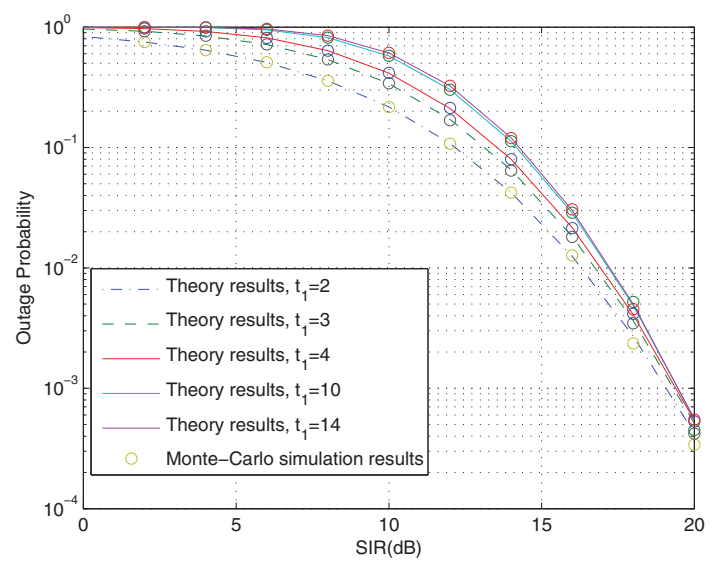

Fig. 5. Variation of outage probability with the number of interfering antennas.

The influence of the interferer's correlation coefficient on the outage probability is shown in Fig 6 where $t_{1}$ is set to 3 and the three curves are shown for $g_{1}=0.3,0.8$ and 0.9 , respectively. Other parameters are set to be $g_{t}=0.5$ and $g_{r}=0.95$. We observe that over the region of moderate and high SIR, the outage performance improves with increased $g_{1}$. This is is easy to understand since a higher interference correlation implies a sharper directional beam 
which is easier to be nullified by using interference-covariance matrix inversion involved in our quadratic form. Clearly, unlike the effect of the intended user's correlation, the spatial correlation of co-channel interference is an advantage to the outage performance of TRD systems. From these curves, we can see, again, a nearly perfect agreement between the theoretical and simulated results.

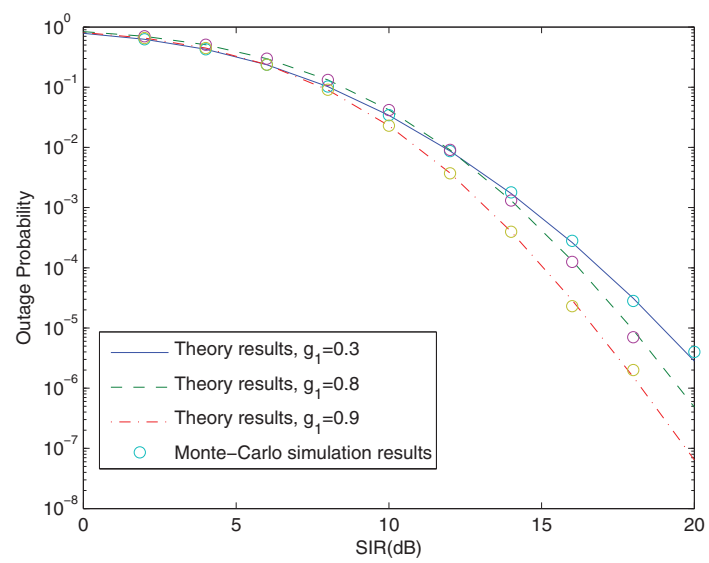

Fig. 6. Influence of interference correlation $g_{1}$ on the outage performance.

In Fig.7, the outage probability versus the number of transmit antennas under different SIRs are plotted. The parameters are set at $r=2, g_{t}=0.5, g_{r}=0.9$ and $g_{1}=0.5$. The three curves in the figure are for $\mathrm{SIR}=10 \mathrm{~dB}, 15 \mathrm{~dB}$ and $20 \mathrm{~dB}$, respectively. As shown in the figure, the outage performances improves almost linearly with the number of transmit antennas $t$ increasing.

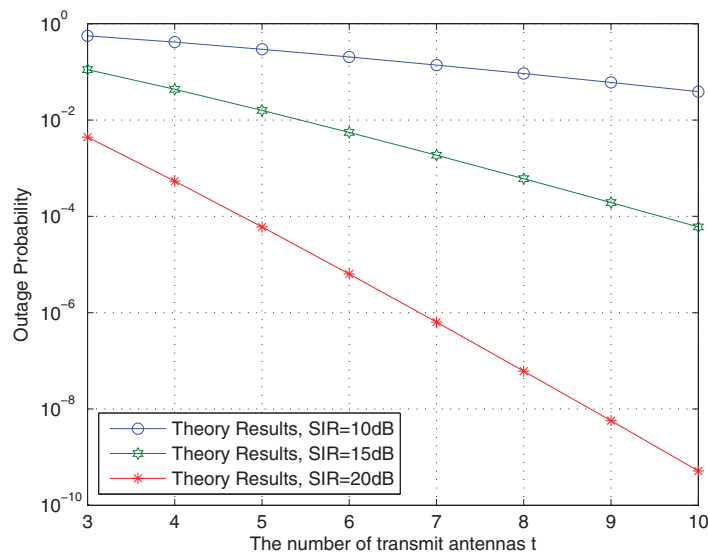

Fig. 7. Influence of signal transmit correlation on the outage probability.

Fig. 8 considers the case when 2 interfering users involved. The 2 interfering channel matrixes are with the same correlation coefficient $g_{1}=0.5$, in the receive end. The equivalent $t_{1}$ and 
$\Sigma_{1}$ are determined by equating the first two moments of $\mathbf{Q}_{1}$ and $\mathbf{R}_{c}$ as we introduced in the previous section. The other parameters are set at $t=3, r=3, g_{t}=0.5$ and $g_{r}=0.9$. We observe the loss of precision as we change the interference power distribution which is denoted by a ratio $\epsilon=E_{1} / E_{2}$. It is shown in the figure that our analysis has high precision when the ratio $\epsilon$ is close to 1 , however, when the ratio loses balance, say $\epsilon=5$, the theory curve can only be considered as a lower bound of the real performance.

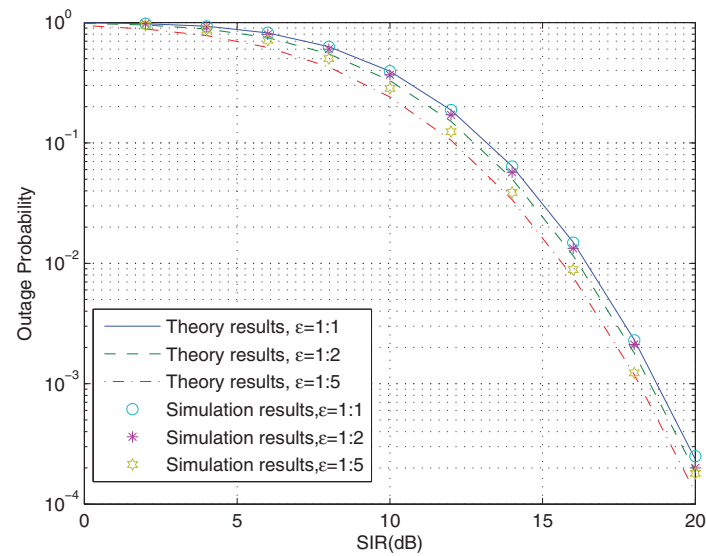

Fig. 8. Influence of the number of transmit antennas on the outage probability.

We next consider the case without co-channel interference. Fig. 9 shows the outage probability as a function of SIR for different values of $g_{t}$. Here we set $g_{r}=0.5$. The three curves are for $g_{t}=0.1,0.5$ and 0.9 , respectively. It is clear that the outage performance drops with increased transmit correlation coefficient $g_{t}$. This is quite intuitive since high transmit correlation means the lose of more degrees of freedom in transmit diversity. A perfect agreement between simulation and theoretic results are observed again.

\section{Conclusions}

Wireless transmission using multiple antennas has attracted much interest due to its capability to exploit the tremendous capacity inherent in MIMO channels. However, the performance of MIMO systems is very sensitive to the presence of co-channel interference or spatial fading correlation. In this chapter, based on the theory of complex matrix variate distributions, we have investigated the performance of MIMO systems in the presence of both co-channel interference and spatial correlation. We first have derived several exact closed-form expressions of the MIMO ergodic capacity in Rayleigh fading environments, and demonstrated by experimentation the influences of co-channel interference and spatial correlation on the ergodic capacity. Then we have tackled the outage performance issue of MIMO systems with optimal transmit/receive diversity, and obtained two formulas of outage probability for general cases of Rayleigh faded signals with and without Rayleigh faded interference, respectively. Finally, we have presented numerical results to validate the theoretical analysis of outage probability. It can been found that the theoretical analysis 


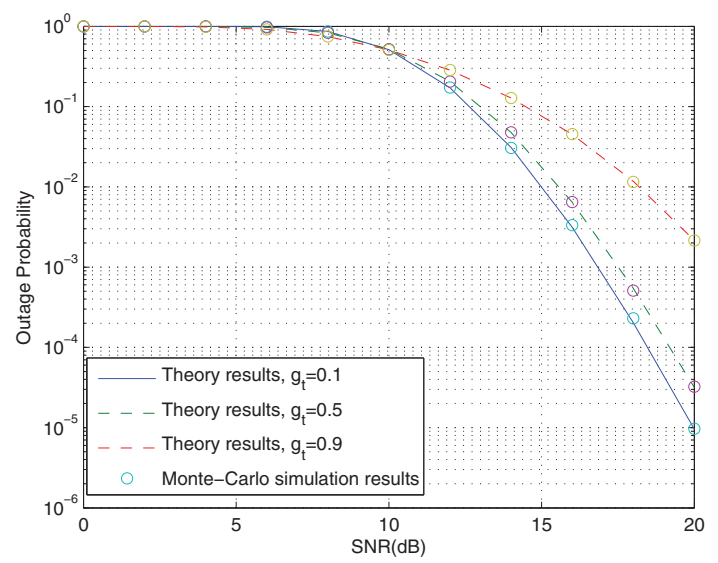

Fig. 9. Influence of the interference power distribution on the outage probability.

of MIMO systems with co-channel interference and spatial correlation depends heavily on multivariate statistics knowledge, especially the theory of matrix variate distributions.

\section{Appendix: Proofs of theorem 1 and theorem 2 in section 3}

\subsection{Proof of theorem 1}

Proof of Theorem 1 : a) Suppose that $t \leq r$. From Equation (61) of [Khatri (1966)], the PDF of the random matrix $\mathbf{Q}$ can be written as

$$
\begin{aligned}
f(\mathbf{Q})= & \frac{\tilde{\Gamma}_{r}\left(t+t_{I}\right)}{\tilde{\Gamma}_{r}\left(t_{I}\right) \tilde{\Gamma}_{t}(r)}\left|\rho \mathbf{I}_{t}\right|^{-r}|\tilde{\boldsymbol{\Sigma}}|^{-t}|\mathbf{Q}|^{r-t} \\
& \left|\mathbf{I}_{t}+(q \rho)^{-1} \mathbf{Q}\right|^{-\left(t+t_{I}\right)}{ }_{1} \tilde{F}_{0}^{(t, r)}\left(t+t_{I}, \mathbf{Q}\left(q \rho \mathbf{I}_{t}+\mathbf{Q}\right)^{-1}, \mathbf{I}_{r}-q \tilde{\boldsymbol{\Sigma}}^{-1}\right)
\end{aligned}
$$

where $q$ is an arbitrary scalar constant. Let $q=\rho^{-1}$. Then we get after simplifying

$$
\begin{aligned}
f(\mathbf{Q})= & \frac{\tilde{\Gamma}_{r}\left(t+t_{I}\right)}{\tilde{\Gamma}_{r}\left(t_{I}\right) \tilde{\Gamma}_{t}(r)}|\rho \tilde{\boldsymbol{\Sigma}}|^{-t}|\mathbf{Q}|^{r-t} \\
& \left|\mathbf{I}_{t}+\mathbf{Q}\right|^{-\left(t+t_{I}\right)}{ }_{1} \tilde{F}_{0}^{(t, r)}\left(t+t_{I}, \mathbf{Q}\left(\mathbf{I}_{t}+\mathbf{Q}\right)^{-1}, \mathbf{I}_{r}-(\rho \tilde{\mathbf{\Sigma}})^{-1}\right)
\end{aligned}
$$

Make the transformation

$$
\mathbf{L}=\left(\mathbf{I}_{t}+\mathbf{Q}\right)^{-1} \mathbf{Q},
$$

and the Jacobian of the transformation is given by Equation (5.1.3) of [Khatri (1965)]

$$
J(\mathbf{Q} ; \mathbf{L})=\left|\mathbf{I}_{t}-\mathbf{L}\right|^{-2 t}
$$

Thus the MGF of mutual information $I(\mathbf{s}, \mathbf{y})$ is expressed as

$$
\begin{aligned}
M(\theta) & =\int_{\mathbf{Q}>0}|\mathbf{I}+\mathbf{Q}|^{\theta} f(\mathbf{Q}) d \mathbf{Q} \\
& =\frac{\tilde{\Gamma}_{r}\left(t+t_{I}\right)}{\tilde{\Gamma}_{r}\left(t_{I}\right) \tilde{\Gamma}_{t}(r)|\rho \tilde{\mathbf{\Sigma}}|^{t}} \int_{0<L<\mathbf{I}_{t}}|\mathbf{L}|^{r-t}|\mathbf{I}-\mathbf{L}|^{t_{I}-r-\theta}{ }_{1} \tilde{F}_{0}^{(t, r)}\left(t+t_{I}, \mathbf{L}, \mathbf{I}_{r}-(\rho \tilde{\mathbf{\Sigma}})^{-1}\right) d \mathbf{L}(100)
\end{aligned}
$$


Using Equation (7) of [Khatri (1966)] and Definition 2 here, we further have

$$
M(\theta)=\frac{\tilde{\Gamma}_{r}\left(t+t_{I}\right) \tilde{\Gamma}_{t}\left(t+t_{I}-r-\theta\right)}{\tilde{\Gamma}_{r}\left(t_{I}\right) \tilde{\Gamma}_{t}\left(t+t_{I}-\theta\right)}|\rho \tilde{\boldsymbol{\Sigma}}|^{-t}{ }_{2} \tilde{F}_{1}^{(t, r)}\left(t+t_{I}, r ; t+t_{I}-\theta ; \mathbf{I}_{t}, \mathbf{I}_{r}-(\rho \tilde{\mathbf{\Sigma}})^{-1}\right) .
$$

From Equation (54) of [Shin \& Lee (2003)] or Property 2 in Section 2, we have

$$
\frac{C_{\kappa}\left(\mathbf{I}_{t}\right)}{C_{\kappa}\left(\mathbf{I}_{r}\right)}=\frac{[t]_{\mathcal{K}}}{[r]_{\mathcal{K}}}
$$

Therefore, we have by noting relationship between the hypergeometric function of two matrix arguments and the hypergeometric function of one matrix argument (involving Property 2 and Property 6)

$$
{ }_{2} \tilde{F}_{1}^{(t, r)}\left(t+t_{I}, r ; t+t_{I}-\theta ; \mathbf{I}_{t}, \mathbf{I}_{r}-(\rho \tilde{\mathbf{\Sigma}})^{-1}\right)={ }_{2} \tilde{F}_{1}^{(r)}\left(t+t_{I}, t ; t+t_{I}-\theta ; \mathbf{I}_{r}-(\rho \tilde{\mathbf{\Sigma}})^{-1}\right)
$$

Applying (49) of James [James (1964)] to the above expression, we further get

$$
\begin{aligned}
M(\theta) & =\frac{\tilde{\Gamma}_{r}\left(t+t_{I}\right) \tilde{\Gamma}_{t}\left(t+t_{I}-r-\theta\right)}{\tilde{\Gamma}_{r}\left(t_{I}\right) \tilde{\Gamma}_{t}\left(t+t_{I}-\theta\right)} \mid \rho \tilde{\boldsymbol{\Sigma}}^{-t}{ }_{2} \tilde{F}_{1}^{(r)}\left(t+t_{I}, t ; t+t_{I}-\theta ; \mathbf{I}_{r}-(\rho \tilde{\boldsymbol{\Sigma}})^{-1}\right) \\
& =\frac{\tilde{\Gamma}_{r}\left(t+t_{I}\right) \tilde{\Gamma}_{t}\left(t+t_{I}-r-\theta\right)}{\tilde{\Gamma}_{r}\left(t_{I}\right) \tilde{\Gamma}_{t}\left(t+t_{I}-\theta\right)}{ }_{2} \tilde{F}_{1}^{(r)}\left(-\theta, t ; t+t_{I}-\theta ; \mathbf{I}_{r}-(\rho \tilde{\boldsymbol{\Sigma}})\right) .
\end{aligned}
$$

It is obvious that

$$
\frac{\tilde{\Gamma}_{t}\left(t+t_{I}-r-\theta\right)}{\tilde{\Gamma}_{t}\left(t+t_{I}-\theta\right)}=\frac{\tilde{\Gamma}_{r}\left(t_{I}-\theta\right)}{\tilde{\Gamma}_{r}\left(t+t_{I}-\theta\right)}
$$

Thus we obtain the desired result

$$
M(\theta)=\frac{\tilde{\Gamma}_{r}\left(t+t_{I}\right) \tilde{\Gamma}_{r}\left(t_{I}-\theta\right)}{\tilde{\Gamma}_{r}\left(t_{I}\right) \tilde{\Gamma}_{r}\left(t+t_{I}-\theta\right)} 2 \tilde{F}_{1}^{(r)}\left(-\theta, t ; t+t_{I}-\theta ; \mathbf{I}-\rho \tilde{\boldsymbol{\Sigma}}\right) .
$$

b) Now we consider the case where $r \leq t$. It follows easily that

$$
|\mathbf{I}+\mathbf{Q}|=|\mathbf{I}+\mathbf{F}|
$$

where $\mathbf{F}=\tilde{\mathbf{R}}^{-1 / 2} \tilde{\mathbf{H}} \tilde{\mathbf{H}}^{\dagger} \tilde{\mathbf{R}}^{-1 / 2}$. In order to get an expression of $M(\theta)$, we can make use of the PDF of the random matrix F to replace the PDF of $\mathbf{Q}$. Based on Equation (62) of [Khatri (1965)], the PDF of the random matrix $\mathbf{F}$ is given by

$$
\begin{aligned}
f(\mathbf{F})= & \frac{\tilde{\Gamma}_{r}\left(t+t_{I}\right)}{\tilde{\Gamma}_{r}\left(t_{I}\right) \tilde{\Gamma}_{r}(t)}|\rho \tilde{\boldsymbol{\Sigma}}|^{-t}|\mathbf{F}|^{t-r} \\
& \cdot\left|\mathbf{I}_{r}+(q \rho \tilde{\boldsymbol{\Sigma}})^{-1} \mathbf{F}\right|^{-\left(t+t_{I}\right)}{ }_{1} \tilde{F}_{0}^{(r, t)}\left(t+t_{I}, \mathbf{F}(q \rho \tilde{\boldsymbol{\Sigma}}+\mathbf{F})^{-1}, \mathbf{I}_{t}-q \mathbf{I}_{t}\right)
\end{aligned}
$$

where $q$ is an arbitrary scalar constant. By taking $q \rightarrow \infty$, the PDF of $\mathbf{F}$ can be rewritten as

$$
f(\mathbf{F})=\frac{\tilde{\Gamma}_{r}\left(t+t_{I}\right)}{\tilde{\Gamma}_{r}\left(t_{I}\right) \tilde{\Gamma}_{r}(t)}|\rho \tilde{\boldsymbol{\Sigma}}|^{-t}|\mathbf{F}|^{t-r}{ }_{1} \tilde{F}_{0}^{(r, t)}\left(t+t_{I}, \mathbf{F}(\rho \tilde{\boldsymbol{\Sigma}})^{-1},-\mathbf{I}_{t}\right)
$$


From Definitions 2 and 3, we obtain with the help of Equation (90) of James [James (1964)]

$$
\begin{aligned}
f(\mathbf{F}) & =\frac{\tilde{\Gamma}_{r}\left(t+t_{I}\right)}{\tilde{\Gamma}_{r}\left(t_{I}\right) \tilde{\Gamma}_{r}(t)}|\rho \tilde{\boldsymbol{\Sigma}}|^{-t}|\mathbf{F}|^{t-r}{ }_{1} \tilde{F}_{0}^{(r)}\left(t+t_{I},\left(\rho \tilde{\mathbf{\Sigma}} \mathbf{I}_{r}\right)^{-1} \mathbf{F}\right) \\
& =\frac{\tilde{\Gamma}_{r}\left(t+t_{I}\right)}{\tilde{\Gamma}_{r}\left(t_{I}\right) \tilde{\Gamma}_{r}(t)}|\rho \tilde{\boldsymbol{\Sigma}}|^{-t}|\mathbf{F}|^{t-r}\left|\mathbf{I}_{r}+\left(\rho \tilde{\boldsymbol{\Sigma}} \mathbf{I}_{r}\right)^{-1} \mathbf{F}\right|^{-\left(t+t_{I}\right)} .
\end{aligned}
$$

Thus the MGF of mutual information $I(\mathbf{s}, \mathbf{y})$ can be expressed as

$$
\begin{aligned}
M(\theta) & =\int_{\mathbf{F}}|\mathbf{I}+\mathbf{F}|^{\theta} f(\mathbf{F}) d \mathbf{F} \\
& =\frac{\tilde{\Gamma}_{r}\left(t+t_{I}\right)}{\tilde{\Gamma}_{r}\left(t_{I}\right) \tilde{\Gamma}_{r}(t)|\rho \tilde{\mathbf{\Sigma}}|^{t}} \int_{\mathbf{F}>0}|\mathbf{F}|^{t-r}\left|\mathbf{I}_{r}+\mathbf{F}\right|^{\theta}\left|\mathbf{I}_{r}+\left(\rho \tilde{\mathbf{\Sigma}} \mathbf{I}_{r}\right)^{-1} \mathbf{F}\right|^{-\left(t+t_{I}\right)} d \mathbf{F} .
\end{aligned}
$$

Using Problem 1.18 of [Gupta \& Nagar (2000)], we get the following desired result with the help of (49) of James [James (1964)]

$$
M(\theta)=\frac{\tilde{\Gamma}_{r}\left(t+t_{I}\right) \tilde{\Gamma}_{r}\left(t_{I}-\theta\right)}{\tilde{\Gamma}_{r}\left(t_{I}\right) \tilde{\Gamma}_{r}\left(t+t_{I}-\theta\right)} 2 \tilde{F}_{1}^{(r)}\left(-\theta, t ; t+t_{I}-\theta ; \mathbf{I}-\rho \tilde{\boldsymbol{\Sigma}}\right) .
$$

\subsection{Proof of theorem 2}

Proof of Theorem 2: By Theorem 1 we get

$$
\begin{aligned}
C= & \left.\log _{2} e \cdot \frac{\partial M(\theta)}{\partial \theta}\right|_{\theta=0} \\
= & \log _{2} e \cdot \frac{\partial}{\partial \theta}\left\{\frac{\tilde{\Gamma}_{r}\left(t+t_{I}\right) \tilde{\Gamma}_{r}\left(t_{I}-\theta\right)}{\tilde{\Gamma}_{r}\left(t_{I}\right) \tilde{\Gamma}_{r}\left(t+t_{I}-\theta\right)} 2 \tilde{F}_{1}^{(r)}\left(-\theta, t ; t+t_{I}-\theta ; \mathbf{I}-\rho \tilde{\mathbf{\Sigma}}\right)\right\} \\
= & \left.\log _{2} e \cdot \frac{\partial}{\partial \theta}\left\{\frac{\tilde{\Gamma}_{r}\left(t+t_{I}\right) \tilde{\Gamma}_{r}\left(t_{I}-\theta\right)}{\tilde{\Gamma}_{r}\left(t_{I}\right) \tilde{\Gamma}_{r}\left(t+t_{I}-\theta\right)}\right\}\right|_{\theta=0}{ }_{2} \tilde{F}_{1}^{(r)}\left(0, t ; t+t_{I} ; \mathbf{I}-\rho \tilde{\mathbf{\Sigma}}\right) \\
& +\left.\log _{2} e \cdot \frac{\partial}{\partial \theta}\left\{{ }_{2} \tilde{F}_{1}^{(r)}\left(-\theta, t ; t+t_{I}-\theta ; \mathbf{I}-\rho \tilde{\mathbf{\Sigma}}\right)\right\}\right|_{\theta=0} \\
= & \log _{2} e(A+B)
\end{aligned}
$$

In what follows, we will derive expressions of $A$ and $B$ in order to compute C. By (87) of James [James (1964)], we can have

$$
{ }_{2} \tilde{F}_{1}^{(r)}\left(0, t ; t+t_{I} ; \mathbf{I}-\rho \tilde{\boldsymbol{\Sigma}}\right)=1 .
$$

For an integer $r \leq a$, we get with the definition of gamma function

$$
\begin{aligned}
\left.\frac{\partial}{\partial \theta} \Gamma_{r}(a-\theta)\right|_{\theta=0} & =\left.\frac{\partial}{\partial \theta} \prod_{i=1}^{r} \Gamma(a-\theta-i+1)\right|_{\theta=0} \\
& =\left.\sum_{k=1}^{r} \prod_{i=1, i \neq k}^{r} \Gamma(a-i+1) \frac{\partial}{\partial \theta} \Gamma_{r}(a-k-\theta+1)\right|_{\theta=0} \\
& =-\Gamma_{r}(a) \sum_{k=1}^{r} \psi(a-k+1)
\end{aligned}
$$


Here $\psi(\cdot)$ is the digamma function defined by (8.360) of [Gradshteyn \& Ryzhik (1994)]

$$
\psi(x)=\frac{\Gamma^{\prime}(x)}{\Gamma(x)} .
$$

With the help of (8.365) in [Gradshteyn \& Ryzhik (1994)], we can have

$$
\begin{aligned}
A & =\left.\frac{\partial}{\partial \theta}\left\{\frac{\Gamma_{r}\left(t_{I}-\theta\right)}{\Gamma_{r}\left(t_{I}\right)}\right\}\right|_{\theta=0}+\left.\frac{\partial}{\partial \theta}\left\{\frac{\Gamma_{r}\left(t+t_{I}\right)}{\Gamma_{r}\left(t+t_{I}-\theta\right)}\right\}\right|_{\theta=0} \\
& =\sum_{k=1}^{r} \psi\left(t+t_{I}-k+1\right)-\sum_{k=1}^{r} \psi\left(t_{I}-k+1\right) \\
& =\sum_{k=1}^{r} \sum_{j=0}^{t-1} \frac{1}{t_{I}-k+1+j}
\end{aligned}
$$

Now we consider how to compute $B$. From Lemma 1 it is known that

$$
{ }_{2} \tilde{F}_{1}^{(r)}\left(-\theta, t ; t+t_{I}-\theta ; \mathbf{I}-\rho \tilde{\boldsymbol{\Sigma}}\right)=\frac{|\mathbf{G}|}{|V(\boldsymbol{\Delta})|}
$$

where $\mathbf{G}=\left[g_{i, j}\right]$ with

$$
g_{i, j}=\delta_{i}^{r-j}{ }_{2} F_{1}\left(-\theta-j+1, t-j+1 ; t+t_{I}-\theta-j+1 ; \delta_{i}\right)
$$

for $i, j=1,2, \ldots, r$. In particular, we get by (3) of James [James (1964)]

$$
\left.g_{i, j}\right|_{\theta=0}=\sum_{k=0}^{j-1} \frac{(-j+1)_{k}(t-j+1)_{k} \delta_{i}^{r-j+k}}{\left(t+t_{I}-j+1\right)_{k} k !} .
$$

a) For $r \leq t$, it follows with the help of (48) of James [James (1964)]

$$
\begin{aligned}
\left.\frac{\partial g_{i, j}}{\partial \theta}\right|_{\theta=0}= & \left.\delta_{i}^{r-j} \frac{\partial}{\partial \theta} \frac{\Gamma\left(t+t_{I}-\theta-j+1\right)}{\Gamma\left(t_{I}-\theta\right) \Gamma(t-j+1)} \int_{0}^{1} x^{t-j}(1-x)^{t_{I}-\theta-1}\left(1-\delta_{i} x\right)^{j-1+\theta} d x\right|_{\theta=0} \\
= & \delta_{i}^{r-j} \frac{\Gamma\left(t+t_{I}-j+1\right)}{\Gamma\left(t_{I}\right) \Gamma(t-j+1)} \int_{0}^{1} x^{t-j}(1-x)^{t_{I}-1}\left(1-\delta_{i} x\right)^{j-1}\left[\ln \left(1-\delta_{i} x\right)-\ln (1-x)\right] d x \\
& +\delta_{i}^{r-j}\left(\psi\left(t_{I}\right)-\psi\left(t_{I}+t-j+1\right)\right)_{2} F_{1}\left(-j+1, t-j+1 ; t+t_{I}-j+1 ; \delta_{i}\right) \\
= & \delta_{i}^{r-j} \frac{\Gamma\left(t+t_{I}-j+1\right)}{\Gamma\left(t_{I}\right) \Gamma(t-j+1)} \int_{0}^{1} x^{t-j}(1-x)^{t_{I}-1}\left(1-\delta_{i} x\right)^{j-1}\left[\ln \left(1-\delta_{i} x\right)-\ln (1-x)\right] d x \\
& -\left(\sum_{b=0}^{t-j} \frac{1}{t_{I}+b}\right) \sum_{k=0}^{j-1} \frac{(-j+1)_{k}(t-j+1)_{k} \delta_{i}^{r-j+k}}{\left(t+t_{I}-j+1\right)_{k} k !} .
\end{aligned}
$$

Therefore, we have when $r \leq t$

$$
\begin{aligned}
B & =\left.\frac{\partial}{\partial \theta}\left\{{ }_{2} \tilde{F}_{1}^{(r)}\left(-\theta, t ; t+t_{I}-\theta ; \mathbf{I}-\rho \tilde{\mathbf{\Sigma}}\right)\right\}\right|_{\theta=0} \\
& =\frac{\sum_{h=1}^{r}|\mathbf{D}(h)|}{|V(\boldsymbol{\Delta})|}
\end{aligned}
$$


where $D(h)=\left(d_{i, j}(h)\right)$ with

$$
d_{i, j}(h)= \begin{cases}\sum_{k=0}^{j-1} \frac{(-j+1)_{k}(t-j+1)_{k} \delta_{i}^{r-j+k}}{\left(t+t_{I}-j+1\right)_{k} k !}, & j \neq h \\ h_{i, j}-\sum_{b=0}^{t-j} \frac{1}{t_{I}+b} \sum_{k=0}^{j-1} \frac{(-j+1)_{k}(t-j+1)_{k} \delta_{i}^{r-j+k}}{\left(t+t_{I}-j+1\right)_{k} k !}, j=h .\end{cases}
$$

Here $h_{i, j}$ is defined by

$$
h_{i, j}=\delta_{i}^{r-j} \frac{\Gamma\left(t+t_{I}-j+1\right)}{\Gamma\left(t_{I}\right) \Gamma(t-j+1)} \int_{0}^{1} x^{t-j}(1-x)^{t_{I}-1}\left(1-\delta_{i} x\right)^{j-1}\left[\ln \left(1-\delta_{i} x\right)-\ln (1-x)\right] d x
$$

b) When $t<r$, we note that for $j>t$

$$
g_{i, j}=\sum_{k=0}^{j-1-t} \frac{(-\theta-j+1)_{k}(t-j+1)_{k} \delta_{i}^{r-j+k}}{\left(t+t_{I}-j+1-\theta\right)_{k} k !} .
$$

After some column operations on the determinant $|G|$, we can have for $t<r$

$$
B=\frac{\sum_{h=1}^{t}|\mathbf{D}(h)|}{|V(\boldsymbol{\Delta})|}
$$

where $D(h)=\left(d_{i, j}(h)\right)$ with

$$
d_{i, j}(h)= \begin{cases}\sum_{k=0}^{j-1} \frac{(-j+1)_{k}(t-j+1)_{k} \delta_{i}^{r-j+k}}{\left(t+t_{I}-j+1\right)_{k} k !}, & j \neq h, j \leq t \\ \delta_{i}^{r-j}, & j \neq h, j>t \\ h_{i, j}-\sum_{b=0}^{t-j} \frac{1}{t_{I}+b} \sum_{k=0}^{j-1} \frac{(-j+1)_{k}(t-j+1)_{k} \delta_{i}^{r-j+k}}{\left(t+t_{I}-j+1\right)_{k} k !}, j=h .\end{cases}
$$

\section{Appendix: Proofs of theorem 3 and theorem 4 in section 4}

\subsection{Proof of theorem 3}

The Distributions of quadratic forms in matrix argument have been investigated extensively by many authors. For more details, the reader is referred to [Gupta \& Nagar (2000)] and [Mathai et al. (1995)]. In order to prove Theorem 3, we first extend a lemma for real data to its complex counterpart to obtain the following.

Lemma 3. Let $\mathbf{X} \sim C N_{m, n}(\mathbf{M}, \boldsymbol{\Sigma} \otimes \mathbf{\Psi}), \boldsymbol{\Sigma}>0, \mathbf{\Psi}>0$ and let $\mathbf{A}$ be a $n \times n$ Hermite positive definite matrix. Then the PDF of quadratic form $\mathbf{S}=\mathbf{X A \mathbf { X } ^ { \dagger }}$ is given by

$$
\begin{aligned}
& f(\mathbf{S})=f \sum_{k=0}^{\infty} \sum_{\mathcal{K}} \frac{1}{k ![n]_{\mathcal{K}}} \times \\
& P_{\mathcal{K}}\left(\boldsymbol{\Sigma}^{-\frac{1}{2}} \mathbf{M} \Psi^{-\frac{1}{2}}\left(\mathbf{I}_{n}-q \mathbf{B}\right)^{-\frac{1}{2}}, \mathbf{B}^{-1}-q \mathbf{I}_{n}, \boldsymbol{\Sigma}^{-\frac{1}{2}} \mathbf{S} \boldsymbol{\Sigma}^{-\frac{1}{2}}\right)
\end{aligned}
$$


where $\kappa$ denotes a partition of $k, q \geq 0, \mathbf{B}=\Psi^{1 / 2} \mathbf{A} \Psi^{1 / 2}, \mathbf{I}_{n}-q \mathbf{B}>0$ and

$$
f=\frac{\operatorname{etr}\left(-q \boldsymbol{\Sigma}^{-1} \mathbf{S}\right)|\mathbf{S}|^{n-m}}{\widetilde{\Gamma}_{m}(n)|\boldsymbol{\Sigma}| n|\mathbf{\Psi}|^{m}|\mathbf{A}|^{m}} \cdot \operatorname{etr}\left[-\boldsymbol{\Sigma}^{-1} \mathbf{M} \Psi^{-1} \mathbf{M}^{\dagger}\right] .
$$

Note that $q$ is an arbitrary scalar constant. The PDF for $q>0$ is called the Wishart type representation, and for $q=0$ is called the power series type representation.

To prove Theorem 3, we also need two properties of the generalized Hermite polynomial with three complex matrix arguments, as described below.

\section{Lemma 4.}

$$
\begin{aligned}
& \int_{\mathbf{S}>0} e \operatorname{tr}[-\mathbf{G S}]|\mathbf{S}|^{q-p} P_{\mathcal{K}}\left(\mathbf{T}, \mathbf{A}, \mathbf{B}^{-1 / 2} \mathbf{S B}^{-1 / 2}\right) d \mathbf{S} \\
= & \widetilde{\Gamma}_{p}(q, \kappa)|\mathbf{G}|^{-q} P_{\mathcal{K}}\left(\mathbf{T}, \mathbf{A}, \mathbf{B}^{-1 / 2} \mathbf{G}^{-1} \mathbf{B}^{-1 / 2}\right)
\end{aligned}
$$

where

$$
\widetilde{\Gamma}_{p}(a, \kappa)=\pi^{p(p-1) / 2} \prod_{i=1}^{p} \Gamma\left(a+k_{i}-i+1\right) .
$$

Lemma 5.

$$
\begin{aligned}
& \int_{0<\mathbf{S}<\mathbf{V}}|\mathbf{S}|^{q-p} P_{\mathcal{K}}\left(\mathbf{T}, \mathbf{A}, \mathbf{B}^{-1 / 2} \mathbf{S} \mathbf{B}^{-1 / 2}\right) d \mathbf{S} \\
& =\frac{\widetilde{\Gamma}_{p}(q, \kappa) \tilde{\Gamma}_{p}(p)}{\widetilde{\Gamma}_{p}(p+q, \kappa)}|\mathbf{V}|^{q} P_{\mathcal{K}}\left(\mathbf{T}, \mathbf{A}, \mathbf{B}^{-1 / 2} \mathbf{V} \mathbf{B}^{-1 / 2}\right)
\end{aligned}
$$

where $\mathbf{V}$ is an arbitrary Hermite positive definite matrix.

Proof of Theorem 3: We begin with the case of $t \leq r$ and determine the PDF of the quadratic form $\mathbf{J}$ in (68). Under the condition of given matrix $\mathbf{Q}_{1}$, by plugging $q=0$ into (128) of Lemma 3 , the conditional PDF of $\mathbf{J}$ can be expressed as

$$
\begin{aligned}
& \left.f(\mathbf{J})\right|_{Q_{1}}=q_{0} \sum_{k=0}^{\infty} \sum_{\mathcal{K}} \frac{1}{k ![r]_{\mathcal{K}}} \times \\
& P_{\mathcal{K}}\left(\Psi^{-\frac{1}{2}} \mathbf{M}^{\dagger} \boldsymbol{\Sigma}^{-\frac{1}{2}}, \boldsymbol{\Sigma}^{-\frac{1}{2}} \mathbf{Q}_{1} \boldsymbol{\Sigma}^{-\frac{1}{2}}, \Psi^{-\frac{1}{2}} \mathbf{J} \Psi^{-\frac{1}{2}}\right)
\end{aligned}
$$

where

$$
q_{0}=\frac{|\mathbf{J}|^{r-t}}{\widetilde{\Gamma}_{t}(r)\left|\Sigma \mathbf{Q}_{1}^{-1}\right| t|\Psi|^{r}} \operatorname{etr}\left[-(\boldsymbol{\Sigma})^{-1} \mathbf{M} \Psi^{-1} \mathbf{M}^{\dagger}\right] .
$$

Then by applying Lemma 4 we carry on the expectation of $\left.f(\mathbf{J})\right|_{Q_{1}}$ with respect to $\mathbf{Q}_{1} \sim$ $C W_{r}\left(t_{1}, \Sigma_{1}\right)$ yielding

$$
\begin{aligned}
& f(\mathbf{J})=q_{1} \sum_{k=0}^{\infty} \sum_{\kappa} \frac{\left[t+t_{1}\right]_{\kappa}}{k ![r]_{\mathcal{K}}} \times \\
& P_{\mathcal{K}}\left(\mathbf{\Psi}^{-\frac{1}{2}} \mathbf{M}^{\dagger} \boldsymbol{\Sigma}^{-\frac{1}{2}}, \boldsymbol{\Sigma}^{-\frac{1}{2}} \boldsymbol{\Sigma}_{1} \boldsymbol{\Sigma}^{-\frac{1}{2}}, \boldsymbol{\Psi}^{-\frac{1}{2}} \mathbf{J} \boldsymbol{\Psi}^{-\frac{1}{2}}\right)
\end{aligned}
$$


where

$$
q_{1}=\frac{|\mathbf{J}|^{r-t} \tilde{\Gamma}_{r}\left(t+t_{1}\right)}{\widetilde{\Gamma}_{t}(r) \widetilde{\Gamma}_{r}\left(t_{1}\right)\left|\Sigma \Sigma_{1}^{-1}\right| t|\Psi|^{r}} \operatorname{tr}\left[-(\Sigma)^{-1} \mathbf{M} \Psi^{-1} \mathbf{M}^{\dagger}\right] .
$$

The desired outage probability is nothing but the integration of $f(\mathbf{J})$ over $\mathbf{J}<x \mathbf{I}$. The integral, however, involves matrix arguments and needs to be simplified. To this end, we invoke a property of the generalized Hermite polynomial, i.e., Lemma 5. By applying this property,setting $\Omega=x \mathbf{I}$, and using the definitions of $\Delta$ and $\Theta$, we complete the proof for this case of $t \leq r$.

We next consider the case of $r<t$. Let

$$
\mathbf{J}_{1}=\mathbf{H}_{1}^{\dagger} \mathbf{H}_{1}
$$

where $\mathbf{H}_{1}=\left\{\mathbf{Q}_{1}^{-1 / 2} \mathbf{H}\right\}^{\dagger}$. Due to the fact

$$
P_{\text {out }}=\operatorname{Pr}\left(\mathbf{J}<x \mathbf{I}_{t}\right)=\operatorname{Pr}\left(\mathbf{J}_{1}<x \mathbf{I}_{r}\right),
$$

then in this case the proof is quite similar to the proof given for the case where $t \leq r$, and so is omitted.

Finally, we need the identity, $\widetilde{\Gamma_{r}}\left(t+t_{1}\right) \tilde{\Gamma_{t}}\left(t+t_{1}-r\right)=\tilde{\Gamma_{r}}\left(t_{1}\right) \tilde{\Gamma_{t}}\left(t+t_{1}\right)$, to give the unified representation of (71).

\subsection{Proof of theorem 4}

The following property of the generalized Hermite polynomial with three complex matrix arguments is useful in the proof.

Lemma 6. For a $p \times q$ random matrix $\mathbf{V} \sim C N\left(\mathbf{0}, \mathbf{I}_{q} \otimes \mathbf{I}_{p}\right)$,

$$
P_{\mathcal{K}}(\mathbf{T}, \mathbf{A}, \mathbf{B})=\mathcal{E}_{\mathbf{V}}\left[C_{\kappa}\left(-\mathbf{B}(\mathbf{V}-\imath \mathbf{T}) \mathbf{A}(\mathbf{V}-\imath \mathbf{T})^{\dagger}\right)\right] .
$$

where $\imath=\sqrt{-1}$.

In [Teletar (1999)], Telatar gave the following useful limiting result for a Wishart-distributed matrix sequence.

Lemma 7. Let $\mathbf{S}_{n} \sim C W_{r}\left(n, \frac{1}{n} \mathbf{I}_{r}\right)$. When $n \rightarrow \infty$, then

$$
\mathbf{S}_{n} \rightarrow \mathbf{I}_{r}
$$

Proof of Theorem 4: Without loss of generality, we can assume from (85) and (86) that $\boldsymbol{\Phi}_{n}=\mathbf{I}$. Under the condition of Theorem 3, we first let $t_{1}=n$ be a variable, and further let $\mathbf{Q}_{1}(n) \sim C W_{r}\left(n, \frac{1}{n} \mathbf{I}_{n}\right)$. Then, according to Lemma 7 , we can assert that when $n \rightarrow \infty$, the TRD system with co-channel interference will reduce to the TRD without co-channel interference. Correspondingly, the outage probability of the optimal TRD system with co-channel interference (71) will reduce to the outage probability of the optimal TRD system without co-channel interference, which is just (87) in Theorem 4 . Let us verify this assertion. By inserting $\Sigma_{1}=\frac{1}{n} \mathbf{I}_{r}$ into (71) and comparing the two expressions of (71) and (87), 
we only need to prove Eqs.(141) and (142) shown below.

a) For $t \leq r$, when $n \rightarrow \infty$, then

$$
\mathbf{P}_{n}=\frac{\widetilde{\Gamma}_{r}(t+n, \kappa)}{n^{r t} \widetilde{\Gamma}_{r}(n)} P_{\mathcal{K}}\left(\mathbf{Y}, \frac{1}{n} \Sigma^{-1}, \mathbf{\Psi}^{-1}\right) \rightarrow P_{\mathcal{K}}\left(\mathbf{Y}, \boldsymbol{\Sigma}^{-1}, \mathbf{\Psi}^{-1}\right) .
$$

b) For $t>r$, when $n \rightarrow \infty$, then

$$
\mathbf{P}_{n}=\frac{\widetilde{\Gamma}_{r}(t+n, \kappa)}{n^{r t} \widetilde{\Gamma}_{r}(n)} P_{\mathcal{K}}\left(\mathbf{Y}, \mathbf{\Psi}^{-1}, \frac{1}{n} \Sigma^{-1}\right) \rightarrow P_{\mathcal{K}}\left(\mathbf{Y}, \mathbf{\Psi}^{-1}, \mathbf{\Sigma}^{-1}\right) .
$$

Here, we have used the fact that

$$
[a]_{\kappa}=\frac{\widetilde{\Gamma}_{m}(a, \kappa)}{\widetilde{\Gamma}_{m}(a)} .
$$

Based on Lemma 6 , the proof of (141) and (142) can be done by showing the validity of the following assertion. Namely, for an arbitrary $r \times r$ Hermite matrix $\mathbf{S}$ and $n \rightarrow \infty$, we have

$$
\mathbf{P}_{n}^{\prime}=\frac{\widetilde{\Gamma}_{r}(t+n, \kappa)}{n^{r t} \widetilde{\Gamma}_{r}(n)} C_{\kappa}\left(\frac{1}{n} \Sigma^{-1} S\right) \rightarrow C_{\kappa}\left(\Sigma^{-1} S\right)
$$

To this end, we invoke Property 1 to simplify (144). It remains to show

$$
\frac{\tilde{\Gamma}_{r}(t+n, \kappa)}{n^{r t+k} \tilde{\Gamma}_{r}(n)} \rightarrow 1
$$

whose validity can be checked by directly using the definition of $\widetilde{\Gamma_{p}}(a, \kappa)$ given in (131).

\section{References}

Telatar I. E.(1999). Capacity of multi-antenna Gaussian channels. European Trans. on Telecomm., Vol. 10, No. 6, Nov.-Dec-1999, pp. 585-596, ISSN 1124-318X.

Foschini. G. J \& Gans. M. J.(1998). On limits of wireless communication in a fading environment when using multiple antennas. Wireless Personal Commun., Vol. 6, Number. 3, Mar- 1998, pp. 311-335, ISSN 1572-834X.

Loyka S.(2001). Channel capacity of MIMO architecture using the exponential correlation matrix. IEEE Commun. Letters, Vol.5, No.9, Sep- 2001, pp.369-371, ISSN 1089-7798.

Chuah C. N., et al(2002). Capacity scaling in MIMO wireless systems under correlated fading. IEEE Trans. Inform. Theory, Vol. 48, No. 3, Mar -2002, pp. 637-650, ISSN 0018-9448.

Chiani M., Win M. Z. \& Zanella A.(2003). On the capacity of spatially correlated MIMO rayleigh-fading channels. IEEE Trans. Inform. Theory, Vol. 49, No. 10, Oct -2003, pp. 2363-2371, ISSN 0018-9448.

Shin H. \& Lee J. H.(2003). Capacity of multiple-antenna fading channels:spatial fading correlation, double scattering, and keyhole. IEEE Trans on Information Tehory, Vol.49, No.10, Oct -2003, pp.2636-2647, ISSN 0018-9448. 
Kiessling M.(2005). Unifying analysis of ergodic MIMO capacity in correlated rayleigh fading enviroments. European Transactions on Telecommunications, Vol.16, No.1, Jan -2005, pp.17-35, ISSN 1124-318X.

Catreux S., Greenstein L. J. \& Dressen P. F.(2000). Simulation results for an interference-limited multiple-input multiple-output cellular system. IEEE Commun. Letters, Vol.4, No. 11, Nov -2000, pp. 334-336, ISSN 1089-7798.

Blum R. S., Winters J. H. \& Sollenberger N. R.(2002). On the capacity of cellular systems with MIMO. IEEE Commun. Letters, Vol. 6, No. 6, Jun -2002, pp. 242-244, ISSN 1089-7798.

Blum R.S.(2003). Analysis of MIMO capacity with interference. In proc. International Conference on communications, 2003. (ICC'03. IEEE), pp.2991-2995, ISBN 0-7803-7802-4, Anchorage, USA, May 2003.

Song Y. \& Blostein S. D.(2002). MIMO Channel Capacity in co-channel interference. in Proc 21st Biennial Symposium on Communications, pp.220-224, ISBN Kingston, Canada, May 2002.

Ye S. \& Blum R. S.(2005). Some properties of the capacity of MIMO systems with co-channel interference in Proc 2005 IEEE International Conference on Acoustics, Speech, and Signal Processing, pp.1153-1156, ISBN 0-7803-8874-7, Philadelphia, PA, USA, March, 2005.

Kang M., Yang L. \& Alouini M. -S(2003). Performance analysis of MIMO channels in presence of co-channel interference and additive Gaussian noise. in Proc. the 35th Annual Conference on Information Sciences and Systems (CISS'2003), Johns Hopkins University, Baltimore, MD, Mar. 2003.

Kang M., Yang L., \& Alouini M.-S.(2007). Capacity of MIMO channel in the presence of co-channel interference. Wireless Communcations and Mobile Computing, vol.7, July -2007, pp.113-125, ISSN 1530-8677.

Kang M. \& Alouini M. -S.(2003). Capacity of MIMO Rician channels with multiple correlated Rayleigh co-channel interferers. in Proc IEEE Global Telecommun. Conf. (Globecom'2003), pp.1119-1123, ISBN 0-7803-7974-8, San Francisco, CA, Dec. 2003.

Wang Y. \& Yue D.-W.(2009). Capacity of MIMO Rayleigh fading channels in the presence of interference and receive correlation. IEEE trans. on Vehicular Technology, Vol.58, No.8, Oct -2009, pp.4398-4405, ISSN 0018-9545.

Dighe P. A., Mallik R. K. \& Jamuar S. R.(2001). Analysis of trasmit-receive diversity in Rayleigh fading. in Proc. IEEE Global Telecom. Conf. (Globecom' 2001), pp. 1132-1136, ISBN 0-7803-7206-9, San Antonio, TX, Nov. 2001.

Kang M. \& Alouini M.-S.(2003). Largest eigenvalue of complex Wishart matrices and performance of MIMO MRC systems. IEEE Journal on Selected Areas in Communications: Special Issue on MIMO Systems and Applications (JSAC-MIMO), Vol. 21, No. 3, Apr -2003, pp. 418-426, ISSN 0733-8716.

Kang M. \& Alouini M.-S.(2004). A comparative study on the performance of MIMO MRC systems with and without co-channel interference. IEEE Trans. Commun., Vol.52, No.8, Aug -2004, pp.1417-1425, ISSN 0090-6778.

Dighe P. A., Mallik R. K., \& S. S. Jamuar S. S.(2003). Analysis of K-transmit dual-receive diversity with cochannel interferers over a Rayleigh fading channel. Wireless Personal Commun., Vol. 25, No. 2, May -2003, pp. 87-100, ISSN 1572-834X.

Kang M. \& Alouini M. -S.(2004). Quadratic forms in complex Gaussian matrices and performance analysis of MIMO systems with co-channel interference. IEEE Trans. Wireless Commun., Vol.3, No.2, Feb -2004, pp.418-431, ISSN 1536-1276. 
Yue D.- W. \& Zhang Q. T.(2010). Generic approach to the performance analysis of correlated transmit/receive diversity MIMO systems with/without co-channel interference. IEEE Trans on Information Tehory, Vol.56, No.3, Mar -2010, pp.1147-1157, ISSN 0018-9448.

Muirhead R. J.(1982). Aspects of Multivariate Statistical Theory, Wiley, ISBN 0471094420, New York, 1982.

Gupta A. K. \& Nagar D. K.(2000). Matrix Variate Distributions, Chapman\& Hall/CRC, ISBN 1-58488-046-5, NewYork, 2000.

Mathai A. M., Provost S. B. \& Hayakawa T.(1995). Bininear Forms and Zonal Polynomials, Spring-verlag, ISBN 0-387-94522-9, NewYork, 1995.

James A. T.(1964). Distributions of matrix variates and latent roots derived from normal samples. Ann. Math. Stat., Vol. 35, No. 2, June -1964, pp. 475-501, ISSN 0003-4851.

Khatri C. G.(1966). On the certain distribution problems based on positive definite quadratic functions in normal vectors Ann. Math. Statist., Vol. 37, No. 2, June -1966, pp.468-479, ISSN 0003-4851.

C.G.Khatri C. G.(1965). Classical statistical analysis based on a certain multivariate comlex Gaussian distribution. Ann. Math. Statist., Vol.36, No. 1, Jan -1965, pp.98-114, ISSN 0003-4851.

Kermoal J. P., Schumacher L., Pedersen L. I., Mogensen P. E. \& Frederiksen F.(2002). A stochastic MIMO radio channel model with experimental validation. IEEE Journal on Selected Areas in Communications, Vol. 20, No 6, June- 2002 pp.1211-1226, ISSN 0733-8716.

Paulraj A., Nabar R. \& Gore D.(2003). Introduction to Space-Time Wireless Communication, Cambrige University Press, ISBN 0521826152, Cambrige, 2003.

Zhang Q. T. \& Cui X. W.(2004). Outage probability for optimum combining of arbitrarily faded signals in the presence of correlated Raleigh interferers. IEEE trans. on Vehicular Technology, Vol.53, No.4, July -2004, pp.1043-1051, ISSN 0018-9545.

Pearson E.S. \& Hartley H. O.(1976) Biometrika Tables for Statisticians, Biometrika Trust, vol.1, p.234, ISBN 085264700X, London, 1976.

Gradshteyn I. S. and I. M. Ryzhik I. M.(1994) Table of Integrals, Series, and Products, 5th ed. Academic Press, ISBN 0122947568, Orlando, FL, 1994. 


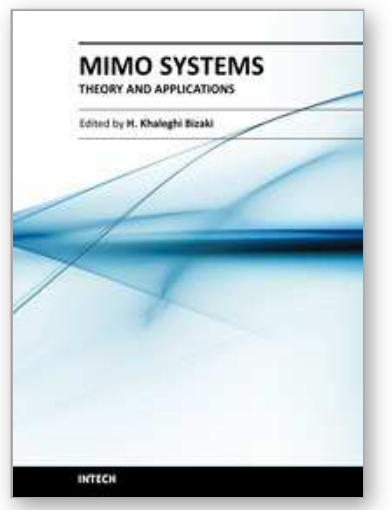

\author{
MIMO Systems, Theory and Applications \\ Edited by Dr. Hossein Khaleghi Bizaki
}

ISBN 978-953-307-245-6

Hard cover, 488 pages

Publisher InTech

Published online 04, April, 2011

Published in print edition April, 2011

In recent years, it was realized that the MIMO communication systems seems to be inevitable in accelerated evolution of high data rates applications due to their potential to dramatically increase the spectral efficiency and simultaneously sending individual information to the corresponding users in wireless systems. This book, intends to provide highlights of the current research topics in the field of MIMO system, to offer a snapshot of the recent advances and major issues faced today by the researchers in the MIMO related areas. The book is written by specialists working in universities and research centers all over the world to cover the fundamental principles and main advanced topics on high data rates wireless communications systems over MIMO channels. Moreover, the book has the advantage of providing a collection of applications that are completely independent and self-contained; thus, the interested reader can choose any chapter and skip to another without losing continuity.

\title{
How to reference
}

In order to correctly reference this scholarly work, feel free to copy and paste the following:

Dian-Wu Yue and Qian Wang (2011). Analysis of MIMO Systems in the Presence of Co-channel Interference and Spatial Correlation, MIMO Systems, Theory and Applications, Dr. Hossein Khaleghi Bizaki (Ed.), ISBN: 978-953-307-245-6, InTech, Available from: http://www.intechopen.com/books/mimo-systems-theory-andapplications/analysis-of-mimo-systems-in-the-presence-of-co-channel-interference-and-spatial-correlation

\section{INTECH}

open science | open minds

\section{InTech Europe}

University Campus STeP Ri

Slavka Krautzeka 83/A

51000 Rijeka, Croatia

Phone: +385 (51) 770447

Fax: +385 (51) 686166

www.intechopen.com

\section{InTech China}

Unit 405, Office Block, Hotel Equatorial Shanghai

No.65, Yan An Road (West), Shanghai, 200040, China 中国上海市延安西路65号上海国际贵都大饭店办公楼 405 单元

Phone: +86-21-62489820

Fax: $+86-21-62489821$ 
(C) 2011 The Author(s). Licensee IntechOpen. This chapter is distributed under the terms of the Creative Commons Attribution-NonCommercialShareAlike-3.0 License, which permits use, distribution and reproduction for non-commercial purposes, provided the original is properly cited and derivative works building on this content are distributed under the same license. 